\title{
In-plane characterization of PZT thin films for the creation of a general impedance model
}

\author{
T. Van de Veire ${ }^{1}$, J. P. George ${ }^{1}$, H. Rijckaert ${ }^{1,2}$, K. Neyts ${ }^{1}$, J. Lauwaert $^{1}$, F. Beunis ${ }^{1}$, J. Beeckman $^{1 *}$ \\ ${ }^{1}$ Department of Electronics \& Information Systems, Ghent University, Technologiepark-Zwijnaarde 126, 9052 \\ Ghent, Belgium \\ ${ }^{2}$ Department of Chemistry, Ghent University, Krijgslaan 281 - S3, 9000 Ghent, Belgium \\ *Corresponding author, jeroen.beeckman@ugent.be
}

\begin{abstract}
The in-plane dielectric and ferroelectric properties of preferentially oriented lead zirconate titanate (PZT) thin films are characterized using interdigitated transducers (IDTs). By combining finite element method simulations and capacitance measurements, values of the dielectric constant of films with thicknesses between $150 \mathrm{~nm}$ and $800 \mathrm{~nm}$ are obtained. A modified Sawyer-Tower circuit is used to investigate the polarization loops measured in-plane using IDT electrodes. A well-defined hysteresis loop is obtained demonstrating the switching of the polarization of the ferroelectric domains. Leakage current measurements reveal high resistivity and are an indication of the high quality of the PZT film. The obtained characteristics are used to determine the total impedance of the IDT-PZT structure. Here, the structure is represented by an equivalent ladder circuit using the inductance and resistance of the IDT electrodes and the capacitance and conductance of the PZT film. The obtained total impedance matches low frequency measurements.
\end{abstract}

\section{Introduction}

Ferroelectric thin films can be found in numerous applications ranging from electro-optic modulation [1], [2], non-volatile memories [3], [4], microelectromechanical systems (MEMS) [5], [6], sensor applications [7] to the generation of surface acoustic waves [8] and even photovoltaics [9]. In many applications interdigitated transducers (IDTs) are used as an aid to convert electrical energy into mechanical energy in the form of Surface Acoustic Waves (SAWs) [10], [11]. Since the electrode structures can be created on top of the ferroelectric film, IDTs have many advantages compared to the use of bottom and top electrodes, such as a larger voltage response since the maximal voltage which can be applied is not limited by the film thickness.

Lead zirconate titanate $\left(\mathrm{PZT}, \mathrm{PbZr}_{1-\mathrm{x}} \mathrm{Ti}_{\mathrm{x}} \mathrm{O}_{3}\right)$ is a ferroelectric material frequently used due to its large dielectric constant, electro-optic and piezoelectric coefficient [12], [13]. Since PZT thin films exhibit excellent piezoelectric properties, they are frequently used in MEMS applications. Additionally, it is a suitable material for acoustic wave devices due to its high electromechanical coupling coefficient [14]. An interesting property of PZT is the existence of the morphotropic phase boundary (MPB) which occurs around $\mathrm{x}=0.48$ [15]. At the MPB, the ferroelectric and piezoelectric properties of PZT are superior and the polycrystalline materials can be switched more easily into a poled state. Unfortunately, PZT thin films do not grow spontaneously in the preferred direction. To grow high-quality thin films on non-lattice matched substrates an intermediate layer is used which acts as a seed layer. In a previous work [16], a novel technique to obtain textured PZT thin films on different substrates using an ultrathin $(5 \mathrm{~nm}-10 \mathrm{~nm})$ dielectric intermediate layer was presented. The ferroelectric films are highly textured with the c-axis perpendicular to the substrate and random in-plane orientation. Previous performed outof-plane measurements indicate excellent electrical performance: a strong remanent polarization, high dielectric constant, low leakage current and high breakdown field are observed.

Accurate determination and prediction of the IDT impedance is indispensable in for example SAW sensor applications [7]. If the pressure, temperature, mass, or liquid on top of the device changes, the 
dielectric properties of the device will change which results in an impedance change that can be measured [17], [18]. Therefore, a characterization of the dielectric properties of the ferroelectric material is necessary for the creation of a general impedance model. Additionally, the model can be used in designing the IDTs in such a way the impedance matches these of the measurement equipment [19].

In this paper, we will discuss the in-plane ferroelectric and dielectric properties, such as dielectric constant, capacitance, polarization and leakage current, of preferentially oriented PZT thin films deposited on glass substrates characterized using IDTs. Since the electric field in between the IDT fingers is no longer linear but curved, the relationship between the capacitance and dielectric constant becomes more complex. In the first part of this paper, the in-plane dielectric constant will be determined by the combination of finite element simulations and capacitance measurements. Thereafter the results will be compared to existing theoretical models [20]-[22]. Additionally, polarization loops are measured in-plane by an extended Sawyer-Tower circuit. Values for the remanent and saturation polarization are obtained together with a value of the dielectric constant of the material. Furthermore, the parasitic resistance of the ferroelectric thin film is obtained from hysteresis measurements at low frequencies [23]. IV-measurements are performed to determine the steady state leakage current, which is different from the leakage current affecting hysteresis loop measurements [24]. Once the PZT layer is fully characterized, a general impedance model of the IDT-PZT device will be discussed and will be compared to measured data.

\section{Experiments}

The PZT (52/48) films are deposited using a chemical solution deposition technique as described in [16]. Preferential orientation onto the Corning Boro-Aluminosilicate glass substrates (Delta Technologies) of the PZT film is obtained using a lanthanide-based seed layer and is verified through XRD measurements. By using a non-conductive substrate, the parasitic capacitance between the electrodes and the substrate can be neglected, which is not the case when using a Si substrate [21]. By repeating the spin coating process, films with thicknesses varying between $150 \mathrm{~nm}$ and $800 \mathrm{~nm}$ are achieved. IDT electrodes consisting of an Au/Ti alloy and a thickness of $400 \mathrm{~nm}$ are developed on top of the PZT film using UV lithography. The spatial wavelength $\left(\lambda_{0}\right)$ of the IDTs varies between 42 and $84 \mu \mathrm{m}$ and the width of the gap and the fingers are equal $\left(\lambda_{0} / 4\right)$.

\section{In-plane characterization of the dielectric constant}

The relation between the in-plane dielectric constant of PZT and the capacitance of an IDT-PZT-glass structure is obtained by combining a FEM simulation in MATLAB and low frequency capacitance C(f) measurements performed using an LCR tool (HP 4192 ALF impedance analyser) with frequencies varying from $1 \mathrm{kHz}$ to $1 \mathrm{MHz}$. Due to the periodicity of the IDT structure, it is sufficient to simulate only one period, consisting of one-half positive and one-half negative electrode, as shown in Figure 1a. The periodicity is implemented by applying Neumann boundary conditions to the left and right edge of the simulation domain. First, the partial differential equation (PDE) solver is used to solve the Laplace equation $\nabla . \epsilon \nabla V=0$ in the structure, after which the electric field can be calculated by $E=-\nabla V$. In the next step, the charge (q) is determined by calculating the Gauss integral along a smooth curve around one half electrode (black line in Figure 1a). Finally, once the charge at one half electrode is known, the capacitance per unit length can be obtained using $\mathrm{c}=\mathrm{q} / \mathrm{V}$ (in $\mathrm{F} / \mathrm{m}$ ). Multiplying $\mathrm{c}$ with $2 \mathrm{~N}-1$, where $\mathrm{N}$ is the number of finger pairs, and the aperture of the IDT (overlap length of the electrode fingers) gives the capacitance (in F) of the total structure. 


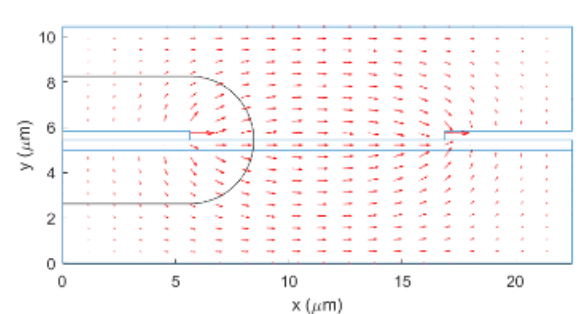

(a)

Figure 1: Simulation result of the electric field of the structure. The PZT layer is a $440 \mathrm{~nm}$ thin layer just below the electrodes, which are modelled as perfect electric conductors. (a) Representation of electric field vectors in the simulated structure, the black line indicates the smooth curve along which the Gauss integral was calculated. (b) Electric field amplitude in the structure.

This simulation is performed for a $440 \mathrm{~nm}$ PZT structure using IDTs with a spatial wavelength $\lambda_{0}$ of 50 or $45 \mu \mathrm{m}$ consisting of 20 finger pairs and an aperture of $36 \lambda_{0}$. Figure 1(b) shows the result of the electric field simulation for $45 \mu \mathrm{m}$ IDTs. Due to the high dielectric constant of the PZT layer, the electric field is quasi-parallel to the PZT layer and almost constant in-between the electrodes. Only near the edges of the electrodes (see Figure 1b) there is a stronger electric field. C(f) measurements of different IDTs with sizes of 50 and $45 \mu \mathrm{m}$ are performed to obtain the dielectric constant. The measured capacitance at 10 $\mathrm{kHz}$ is $25 \mathrm{pF}$ and $25.5 \mathrm{pF}$ for the 50 and $45 \mu \mathrm{m}$ IDTs, respectively. Comparing this to the simulated relation between the dielectric constant and capacitance gives an in-plane dielectric constant of about 1000 .

The same process of simulations and capacitance measurements is performed in order to obtain the inplane dielectric constant for films with thicknesses varying between 150 and 800 nm, shown in Error! Reference source not found.. As this figure indicates, the dielectric constant decreases with increasing thickness of the PZT layer and values between 400 and 2000 are obtained. The large variation of the dielectric constant is probably caused by a deterioration of the film quality of the thicker layers [25]. XRD measurements presented in figure 2(b) indicate excellent preferentially oriented films, irrespective of the layer thickness. XRD measurements are performed using a D8 Discover diffractometer (Bruker technologies Ltd.,) with $\mathrm{CuK} \alpha$ radiation. The peaks in the XRD graph are the (001) and (002) lines of PZT. However, the difference in lattice constant of PZT for the (001) and (100) direction is quite small. As a result, the (001) and (100) lines are very close to each other and this cannot be resolved by the XRD measurements in Figure 2(b). This means that the peaks visible in Figure 2(b) may also be the (100)/(200) peaks of PZT. It is our assumption that a virgin PZT film consists of a mixture of (100) and (001) oriented domains. Thicker films are obtained by the repetition of the spin coating process (one cycle deposits about $50 \mathrm{~nm}$ PZT), which may result in a reduction of the film quality [26] although, even the thicker films do not indicate any crack formation.

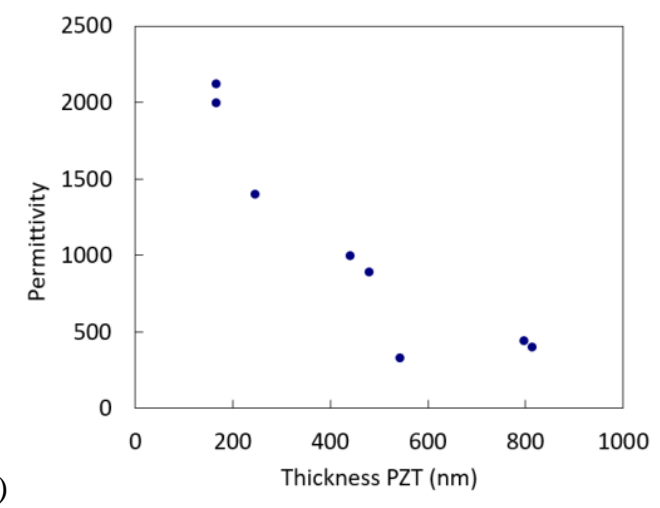

(b)

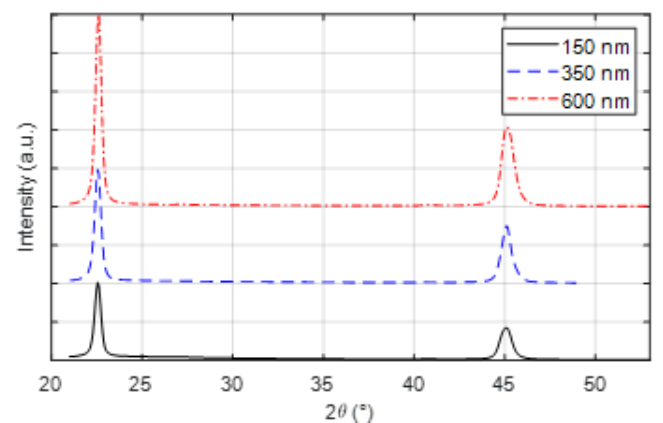


(c)

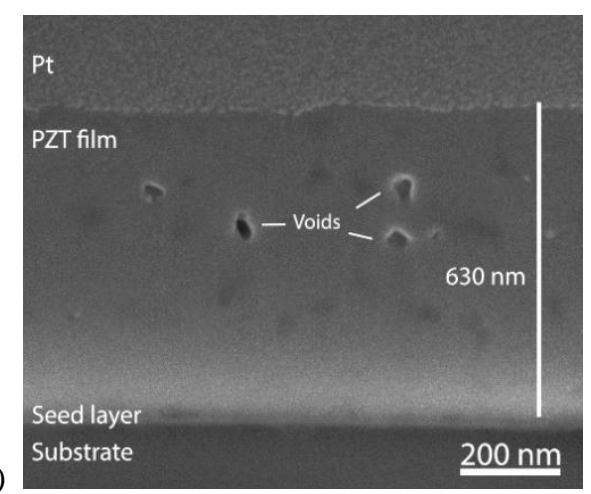

Figure 2: Dielectric constant obtained by combining $C(f)$ measurements with FEM simulations for different thicknesses of the PZT layer (a). XRD measurements for samples with different film thickness $(b)$. Cross-sectional SEM image of PZT film on corning glass substrate (c).

To further investigate the decrease of permittivity in thicker PZT layers, a cross-section of a thick PZT film was performed by first making a cross section using a Focused Ion Beam (FIB) (FEI Nova 600 Nanolab Dual-Beam FIB) and then Scanning Electron Microscopy (SEM) was used to determine the thickness from the cross-sectional views and to study the morphology of PZT film. The PZT layer with a thickness of $630 \mathrm{~nm}$ (shown in Figure 2(c)) is fairly dense, but voids can be detected. It is a wellknown effect that when going towards thicker layers the chance of voids appearing is becoming larger. In this work, this is most probably due to gas release during the thermal processes. Also from a thickness of $250 \mathrm{~nm}$ and higher secondary phases appear (seen as the darker spots in Figure 2(b)). At this moment we do not know yet which exact phase this is as we did not yet perform extensive XRD analysis to identify this phase. It is also well known that the formation of secondary phases is becoming more likely when moving towards thicker layers. Both voids and secondary phases reduce the quality of the PZT layer and subsequently result in lower permittivity. The optimization of the deposition process for obtaining higher quality thick PZT layer is part of future research and is out of the scope of this article.

For the hysteresis measurements, the relation between the geometrical and material parameters and the charge is required to calculate the electric displacement field [27]. This can be achieved by interpolating the results of a limited set of simulations or by using approximate analytical models. Multiple analytical descriptions exist for the relation between the capacitance and dielectric constant, taking the electric field lines into account [20], [21], [28]. The simplest description is the parallel-plate electrode (PPE) model which extends the electrodes through the ferroelectric thin film. The capacitance per unit finger length is then given by

$$
c=\frac{\epsilon_{0} \epsilon_{r} t_{f}}{a}
$$

where $\varepsilon_{\mathrm{r}}$ is the dielectric constant of PZT, $\mathrm{t}_{\mathrm{f}}$ is the PZT film thickness and a is the distance between the electrodes.

More extended descriptions are given by the Gevorgian and Igreja model [20], [21], which also take the stray fields at the end of the fingers and first and last finger into account. It is assumed that the PZT thin film is homogeneous and isotropic, and the presence of the dielectric anisotropy and domain walls is negligible [20]. If the electrode width and gap are much larger than the ferroelectric film thickness, the capacitance per unit length for both models is given by [21]

$$
c=\frac{\epsilon_{0} \epsilon_{r} t_{f}}{a+\Delta a}
$$

where for the Gevorgian capacitance model (full calculation given by Nigon et al. [20])

$$
\Delta a_{G}=\frac{4 \ln (2 \sqrt{2}) t_{f}}{\pi}
$$


and for the Igreja model (full calculation given by Nguyen et al. [21])

$$
\Delta a_{I}=\frac{4 \ln (2) t_{f}}{\pi}
$$

The PPE, Gevorgian and Igreja models can be compared to the results of the FEM simulation for the capacitance and dielectric constant relation for $45 \mu \mathrm{m}$ IDTs and $440 \mathrm{~nm}$ PZT on glass, as shown in Figure 3a. For these relatively large structures and thin films, the PPE model gives the best estimation of the capacitance-dielectric constant relation. This differs from the electric field calculation inside the PZT layer between the finger electrodes where the PPE model overestimates the electric field and the Igreja model is more suited (see Figure 3b). It is possible that the PPE model gives a better estimation to the simulation since for larger gaps and thinner films the Igreja model underestimates the capacitance, as is discussed by Chidambaram [28]. For smaller IDT structures, the Igreja and Gevorgian model are a closer estimation of the simulation. Furthermore, capacitance measurements of IDTs on glass (without a PZT film) are in agreement to simulation results for the same structure, which proves that any parasitic influence of the electrodes can be neglected.

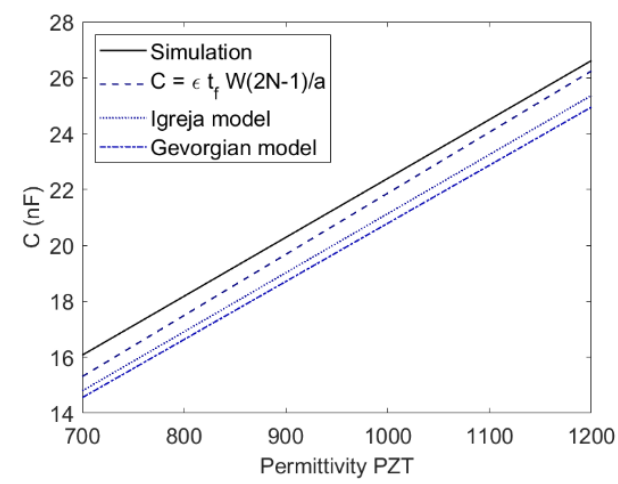

(a)

Figure 3: (a) Comparison of the simulated capacitance-dielectric constant relation with the different theoretical descriptions. (b) Simulated electric field for different voltages applied to $45 \mu \mathrm{m}$ IDTs compared to the electric field calculated using the PPE and Igreja model.

\section{In-plane hysteresis measurements}

To measure the hysteresis loop a modified Sawyer-Tower circuit is used, consisting of a triangular AC voltage source and a known standard capacitor $\left(\mathrm{C}_{0}\right)$ in series with the IDT-PZT-glass sample (device under test, DUT). The measurements are performed using a RS Pro RSDG2000X signal generator combined with a 100x amplifier and a Tektronix TDS7104 oscilloscope which are both connected to a computer and operated by a LabVIEW program. The oscilloscope is used to measure the input voltage $\left(\mathrm{V}_{\text {in }}\right)$ and voltage across the standard capacitor $\left(\mathrm{V}_{0}\right)$. Knowing the voltage across the DUT $\left(\mathrm{V}_{\text {in }}-\mathrm{V}_{0}\right)$ and the distance between the electrodes, the electric field can be calculated using the Igreja model (as is discussed in the previous section). The electric displacement field is obtained by assuming the instantaneous charge stored by the DUT $\left(\mathrm{Q}_{\text {DUT }}=\mathrm{D}(\mathrm{t}) \mathrm{A}_{\mathrm{DUT}}\right)$ and the reference capacitor $\left(\mathrm{Q}_{\mathrm{c}}=\mathrm{C}_{0} \mathrm{~V}_{0}(\mathrm{t})\right)$ are equal:

$$
D(t)=\frac{C_{0}}{A_{D U T}} V_{0}(t)
$$

Since the IDT electrode can be seen as multiple PPE's in parallel, the thickness of the capacitor is equal to the gap between the electrodes and the area $A_{\text {DUT }}=t_{f} W(2 N-1)$, where $\mathrm{W}$ is the aperture length of a single IDT electrode and $\mathrm{N}$ the number of electrode finger pairs. 
Different hysteresis measurements are performed using varying IDT sizes and layer thicknesses, $\mathrm{C}_{0}$ values, amplitudes and frequencies of the AC signal. Figure 4a shows the obtained D-E and P-E loop measured using $50 \mu \mathrm{m}$ IDTs and a $440 \mathrm{~nm}$ thick PZT layer. The frequency and amplitude of the triangular AC signal are $10 \mathrm{kHz}$ and $200 \mathrm{~V}$, respectively. The value of the capacitor in series $\left(\mathrm{C}_{0}\right)$ is 2 $\mathrm{nF}$, which is about 100 times larger than the capacitance value of the PZT device. At first the D-E loop was calculated (light blue curve in Figure 4a). Next a line was fitted to the linear curve at large electric fields to obtain the dielectric constant. The obtained value of the dielectric constant is 1055, which is in agreement with the results of the capacitance and dielectric constant simulation. The ferroelectric part $\mathrm{P}(\mathrm{t})$ of the instantaneous total polarization $\mathrm{P}_{\text {tot }}(\mathrm{t})$ due to the domain switching is then obtained from

$$
D(t)=\epsilon_{0} \epsilon_{P Z T} E(t)+P(t)
$$

and the P-E loop can be plotted (dark blue curve in Figure 4a). The remanent polarization is about 19.5 $\mu \mathrm{C} / \mathrm{cm}^{2}$ and the spontaneous polarization is about $24 \mu \mathrm{C} / \mathrm{cm}^{2}$, where the small difference between these values indicates that the shape of the loop is close to a square. The coercive field is about $45 \mathrm{kV} / \mathrm{cm}$. For PZT films with a smaller thickness, the spontaneous and remanent polarization values are similar to the $440 \mathrm{~nm}$ sample but the coercive field is much smaller, as shown in Figure 4c. These values can be compared to the out-of-plane measurements performed before using $600 \mathrm{~nm}$ thick PZT films of the same composition [16] where a remanent polarization of $19 \mu \mathrm{C} / \mathrm{m}^{2}$ and a coercive field of $68 \mathrm{kV} / \mathrm{cm}$ were measured. The samples in [16] were fabricated using the same deposition procedure and showed the same crystallographic properties as the current samples as confirmed by XRD. The difference is that the glass substrate is coated with an Indium Tin Oxide (ITO) layer before PZT deposition to enable out-ofplane electric fields to be applied. The similarity of these results indicate that the films can be poled equally well in-plane and out-of-plane. We believe that the PZT film goes from an initial mixed $(100) /(001)$ orientation to a dominant (100) orientation in the case of in-plane poling or a dominant (001) orientation in the case of out-of-plane poling.

Additionally, hysteresis measurements are performed using different sizes of the standard capacitor using a frequency of $1 \mathrm{kHz}$. Since most measured hysteresis loops are not fully symmetric but shifted to the right (indicating a preferential polarization direction [29]) and up or down, the values for $2 \mathrm{P}_{\mathrm{r}}, 2$ $P_{s}$ and $2 E_{c}$ are plotted in Figure $4 \mathrm{~b}$. As this figure shows, the size of the standard capacitor has to be at large enough so the voltage over the DUT is large enough to obtain maximal polarization [27].

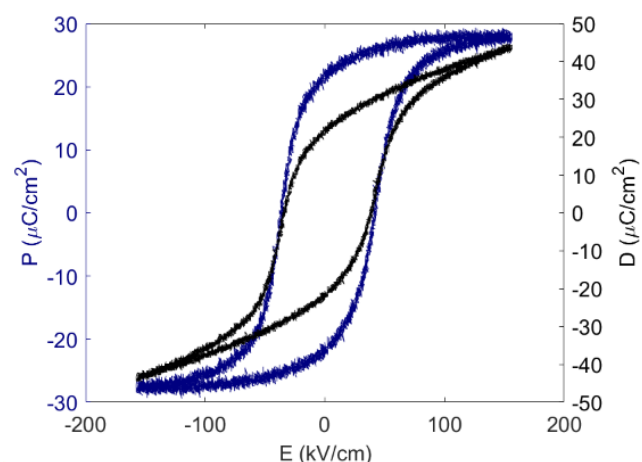

(a)

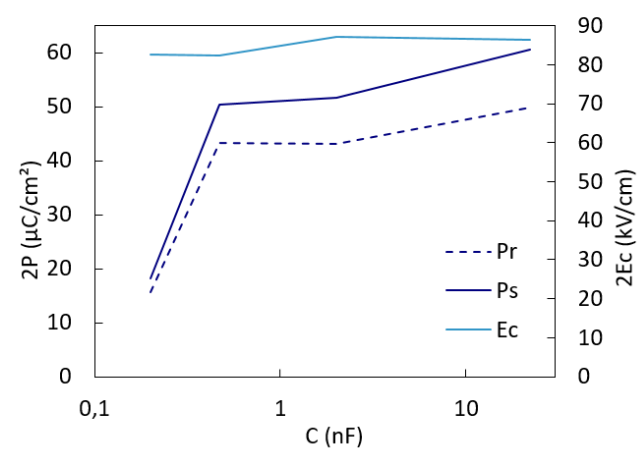

(b) 


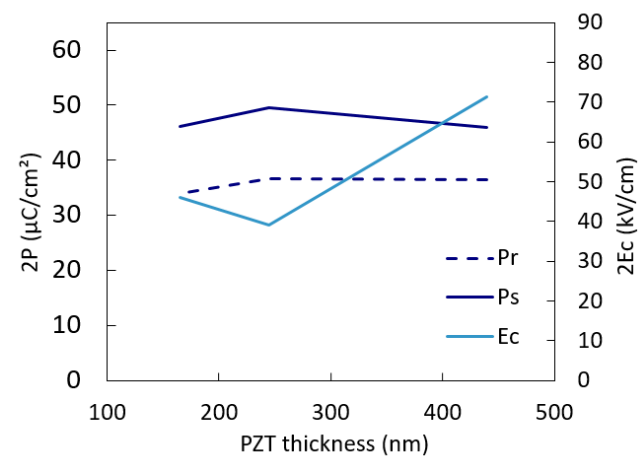

(c)
Figure 4: (a) In-plane measured P-E and D-E loop using $50 \mu \mathrm{m}$ IDTs on a $440 \mathrm{~nm}$ thick PZT layer measured at $10 \mathrm{kHz}$. (b) hysteresis characteristics $\left(2 P_{r}, 2 P_{s}\right.$ and $\left.2 E_{c}\right)$ for different sizes of the standard capacitor $\left(C_{D U T}=25 \mathrm{pF}\right)$ measured at $1 \mathrm{kHz}$ and $(\mathrm{c})$ for different thicknesses of the PZT layer measured at $1 \mathrm{kHz}$ using a $2 \mathrm{nF}$ standard capacitor.

At the metal-PZT interface a Schottky junction will be formed. The study of band alignment between ferroelectric materials is complicated as the polarization may influence the band bending. Thanks to Xray photoelectron spectroscopy (XPS) it is possible to characterize the band bending due to the Schottky mechanism. The band bending at PZT interfaces is in the order of 1.09 to $1.37 \mathrm{eV}$ with a depletion layer of $3 \mathrm{~nm}$ to $35 \mathrm{~nm}$, for metal contacts like Pt, ITO or $\mathrm{SrRuO}_{3}$ [30], [31].XPS analysis of Au contacts on $\mathrm{BaTiO}_{3}$ revealed an apparent built-in potential of $0.3 \mathrm{eV}$ and a depletion width of $6 \mathrm{~nm}$ [32]. The apparent built-in potential for Au contacts on PZT is similar $(0.28 \mathrm{~V})$ but with a much smaller depletion width of about $2 \mathrm{~nm}$ [33]. Due to the fact that the dimensions of the electrode gap (12.5 $\mu \mathrm{m}$ or 11.25 $\mu \mathrm{m})$ are much larger than depletion width and due to the fact that the required voltages for obtaining the hysteresis loop (in the order of $200 \mathrm{~V}$ ) are much larger than the apparent built-in potential, we do not expect any appreciable influence of the Schottky barrier on the measurement results.

\section{Leakage current}

Leakage current measurements are performed on the $440 \mathrm{~nm}$ thick PZT sample using both the $50 \mu \mathrm{m}$ and $45 \mu \mathrm{m}$ IDTs. Two different voltage sweeps are used during the IV measurements: the first one is a step sweep where the voltage alters from $0 \mathrm{~V} \rightarrow+\mathrm{x} \mathrm{V} \rightarrow 0 \mathrm{~V} \rightarrow-\mathrm{x} \mathrm{V}$, where $\mathrm{x}$ varies between $1 \mathrm{~V}$ and $120 \mathrm{~V}$ and the second one is a staircase sweep where the voltage goes from $0 \mathrm{~V}$ to $120 \mathrm{~V}$, then from 120 $\mathrm{V}$ to $-120 \mathrm{~V}$ and finally from $-120 \mathrm{~V}$ to $0 \mathrm{~V}$ in steps of $5 \mathrm{~V}$. During both sweeps the voltage was applied for $10 \mathrm{~s}$ and the current was measured. Figure 5 shows the results of the $50 \mu \mathrm{m}$ IDT for the 2 different sweeps (other measurements give similar results). Each point on the graph represents the current obtained $10 \mathrm{~s}$ after applying the DC voltage. As this figure shows, the leakage remains below $100 \mathrm{pA}$ which is very small and close to the noise region of the detector and the obtained resistivity is about $2 \mathrm{~T} \Omega$. This indicates the high quality of the PZT layer.

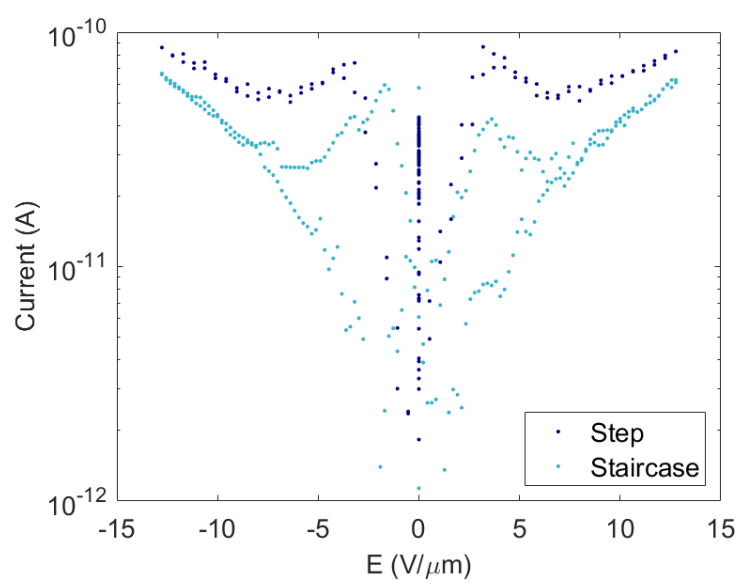

Figure 5: IV measurements of $50 \mu \mathrm{m}$ IDTs up to 120 V measured using a staircase sweep and a step sweep 
The DC leakage current measured is different from the one affecting the hysteresis loops. In the latter, the short measurement time can cause current transients [24]. It is possible to estimate the leakage current during hysteresis measurements. At low frequencies, the P-E loop can be distorted as the result of a phase shift between the applied voltage and measured output voltage caused by the finite conductivity of the ferroelectric sample [34]. If the PZT layer is sufficiently insulating the measured PE loop can be twisted, whereas for samples with a lower resistivity the loop can be distended [23]. This implies that the charge on the electrodes of the DUT and standard capacitor are no longer equal and (5 is not valid anymore. The finite resistance of the ferroelectric sample can be taken into account by placing a resistor $\mathrm{R}_{\mathrm{PZT}}$ in parallel with the DUT. The relation between the charge on the two components is then given by

$$
Q_{D U T}+\int_{(t)} \frac{V_{i n}(t)-V_{0}(t)}{R_{P Z T}} d t=Q_{c}
$$

Combining ( 6 and $(7$ together with the instantaneous charge equations, the polarization can be calculated as

$$
P(t)=\left(\frac{C_{0}}{A_{D U T}}+\frac{\epsilon_{0} \epsilon_{P Z T}}{a+\Delta a}\right) V_{0}(t)-\frac{\epsilon_{0} \epsilon_{P Z T}}{a+\Delta a} V_{i n}(t)-\frac{1}{A_{D U T}} \int_{(t)} \frac{V_{i n}(t)-V_{0}(t)}{R_{P Z T}} d t
$$

Measurements performed at $10 \mathrm{~Hz}$, using $50 \mu \mathrm{m}$ IDTs on a $440 \mathrm{~nm}$ thick PZT film, show indeed a twisted hysteresis loop, as is displayed in Figure 6. Using ( 8 with a resistance value of the PZT equal to $620 \mathrm{M} \Omega$, the twisted P-E loop becomes an untwisted hysteresis loop which has the same saturation polarization as the one measured at $1 \mathrm{kHz}$. However, the remanent polarization and coercive field are smaller compared to the measurements performed at larger frequencies. The relatively high value of the resistance of the PZT obtained from the low frequency hysteresis measurements demonstrates the insulating properties of the PZT layer, which was also observed during DC measurements. Although the leakage current at these higher frequencies is larger compared to the steady state one as the result of current transients due to the short measurement times caused by charges that may not have enough time to settle after polarization switching [24].

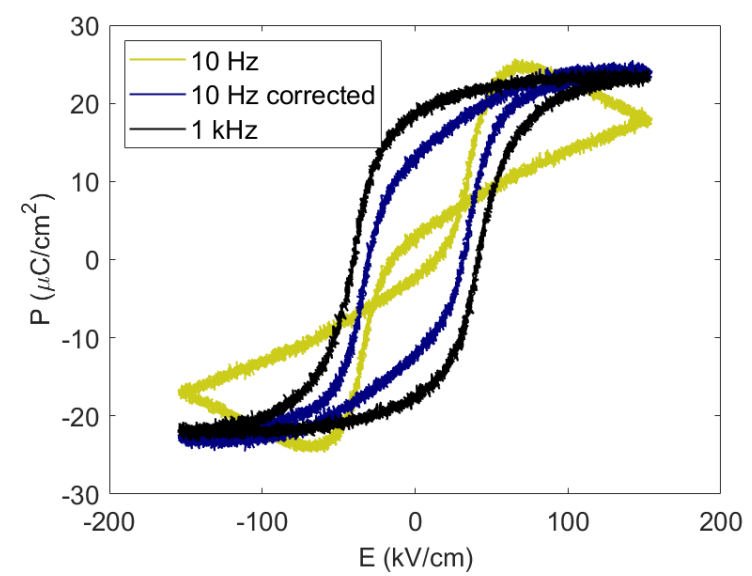

Figure 6: Twisted P-E loop measured at low frequency $(10 \mathrm{~Hz})$ can be corrected to the light blue curve by taking the finite resistivity of the ferroelectric layer into account.

\section{Impedance model}

The total impedance of the IDT-PZT structure using a glass substrate is determined by a quasi-static model. In this model, the electrodes of the IDT are represented by a resistance and inductance and the effect of the PZT layer in between the IDT fingers is replaced by a conductance and capacitance. The total impedance of the IDT-PZT device can be obtained using an equivalent ladder network. 


\section{IV model IDT-PZT structure and voltage along an IDT finger \\ Representation of the PZT layer}

As discussed before, the PZT-IDT structure can be seen as a set of capacitors in parallel. Assuming the electrodes are perfect conductors, only the PZT layer will influence the voltage along the electrodes. Capacitance simulations indicate the capacitance p.u.l. of one finger pair can be represented as if this were a PPE electrode. Beside the capacitance there is also a conductance contribution due to the losses of the PZT layer: there is the dielectric loss which is frequency dependent and given by

$$
g^{\prime}=\omega \epsilon_{I} c_{0}=\frac{\omega \epsilon_{0} \epsilon_{P Z T} \tan \delta t_{f}}{a}
$$

where $\epsilon_{I}$ is the complex part of the permittivity which is obtained from the dielectric loss measurements $\tan \delta=\frac{\epsilon_{P Z T}}{\epsilon_{I}}$ which is frequency dependent, however measurements have shown that in the chosen frequency range the value is almost constant and about 0.02 (see [16]). The second contribution is the leakage current represented by the resistance of the PZT achieved from hysteresis measurements at low frequencies. This makes the total conductance $g=g^{\prime}+\frac{1}{R_{P Z T}}$.

If one assumes perfect electrodes, the voltage-current relation for periodic signals for the 2 electrodes (see Figure 7) is given by

$$
I_{1}=I_{2}=(j \omega c+g)\left(V_{1}-V_{2}\right)=Y_{P Z T}\left(V_{1}-V_{2}\right)
$$

\section{Influence non-perfect electrodes}

Using Ohm's law the current-voltage relation in the electrode fingers due to finite conductivity is given by (see Figure 7)

$$
\begin{aligned}
& I_{1}=-\sigma_{c} d_{c} L_{c} \frac{d V_{1}}{d y} \\
& I_{2}=-\sigma_{c} d_{c} L_{c} \frac{d V_{2}}{d y}
\end{aligned}
$$

where $\sigma_{c}$ is the conductivity, $\mathrm{d}_{\mathrm{c}}$ the thickness and $\mathrm{L}_{\mathrm{c}}$ the length of the electrodes. Due to the capacity of the PZT film, charge conservation states that the surface charge density $\left(K_{x}=Y_{P Z T}\left(V_{1}-V_{2}\right)\right)$ will be conserved between the two finger electrodes:

$$
\begin{gathered}
\frac{d I_{1}}{d y}=-K_{x} \\
\frac{d I_{2}}{d y}=K_{x}
\end{gathered}
$$

Combining (11-(14 results in two coupled differential equations

$$
\begin{gathered}
\frac{d^{2} V_{1}}{d y^{2}}=r_{e l} Y_{P Z T}\left(V_{1}-V_{2}\right) \\
\frac{d^{2} V_{2}}{d y^{2}}=-r_{e l} Y_{P Z T}\left(V_{1}-V_{2}\right)
\end{gathered}
$$

where $r_{e l}=\frac{1}{\sigma_{c} d_{c} L_{c}}$ is the p.u.l. resistance.

Next to the resistance of the electrodes, there will also be an inductance contribution as a result of the magnetic field induced by the current along the electrodes. The inductance contribution exists of both an internal inductance term $\left(\mathrm{L}_{\mathrm{in}}\right)$ and external inductance $\left(\mathrm{L}_{\mathrm{ex}}\right)$, where the last one has only a physical meaning if there are at least two electrodes [35]. 
In contrast to the internal inductance of a round wire, which is well known [36], the internal inductance of an electrode which has a rectangular cross-section is more complex and can no longer be calculated analytically. Therefore, the internal inductance is calculated using a MATLAB simulation where the PDE toolbox is used to calculate the magnetic vector potential inside the electrode [37]

$$
-\nabla^{2} A_{y}(x, y)+j \mu_{0} \sigma \omega A_{y(x, z)}=\mu_{0} J_{a p l}
$$

and applying Dirichlet boundary conditions at the four edges. The current is defined along the y axis hence the simulation can be defined as a $2 \mathrm{D}$ problem. Once the magnetic vector potential is known, the magnetic field is obtained by $B=\nabla \times A_{y} 1_{y}=\nabla A_{y} \times 1_{y}$ and the p.u.l. internal inductance can be calculated as

$$
l_{\text {in }}=\frac{\int|\mathrm{B}|^{2} d S}{\mu_{0}|I|^{2}}
$$

The p.u.l. external inductance for very thin electrodes depends only on the width and distance between the electrodes and is given by [38]

$$
l_{e x}=\frac{\mu_{0} K(k)}{K\left(\sqrt{1-k^{2}}\right)}
$$

where $k=\frac{\frac{a}{L_{C}}}{2+\frac{a}{L_{C}}}$ and $\mathrm{K}$ is the complete elliptic integral of the first kind.

At low frequencies, both the resistance and internal inductance are frequency independent as the current is uniformly distributed across the conductor. For larger frequencies, the skin depth becomes important since the current density will be larger near the surface of the conductor and smaller towards the centre. At a certain frequency, the skin depth will become smaller than the dimensions of the conductor. The frequency at which this occurs is defined as the break-point and depends on the geometry and material of the electrodes [37]. For these high frequencies, the internal inductance will vanish due to the skin effect [39] and the resistance increases with the square root of the frequency $r_{e l}=\frac{1}{2 \sigma_{c} \delta(L c+d c)}$ where $\delta=\sqrt{\left(\frac{2}{\omega \sigma_{c} \mu}\right)}$ is the skin depth. In our designs, the breakpoint is expected to occur at $150 \mathrm{GHz}$, as shown in Figure 8. This frequency is higher than our experimental setup can reach (order of $100 \mathrm{MHz}$ ) and for these frequencies, the resistance can thus be assumed to be frequency independent.

Including the inductance contribution, the two coupled differential equations (15 and 16) for the voltage along the IDT fingers of the network shown in Figure 7 are

$$
\begin{gathered}
\left.\frac{d^{2} V_{1}}{d y^{2}}=r_{e l}+j \omega\left(l_{\text {in }}+l_{e x t}\right)\right) Y_{P Z T}\left(V_{1}-V_{2}\right)=Z_{e l} Y_{P Z T}\left(V_{1}-V_{2}\right) \\
\frac{d^{2} V_{2}}{d y^{2}}=-\left(r_{e l}+j \omega\left(l_{\text {in }}+l_{\text {ext }}\right)\right) Y_{P Z T}\left(V_{1}-V_{2}\right)=-Z_{e l} Y_{P Z T}\left(V_{1}-V_{2}\right)
\end{gathered}
$$

These equations are similar to the Telegrapher's equations for a transmission line, but the boundary conditions are different from these of transmission lines, as will be discussed in the next section. 


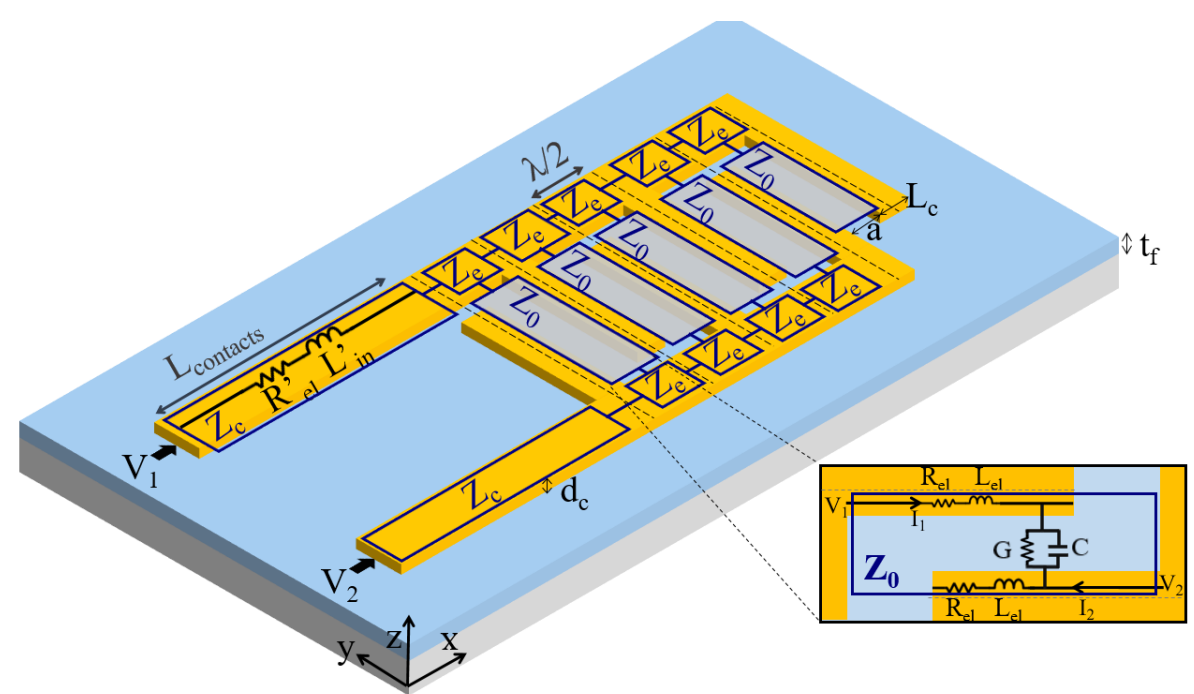

Figure 7: Schematic representation of the different parameters, impedances and network representation of the IDT-PZT structure, including the ladder network representation of the device.

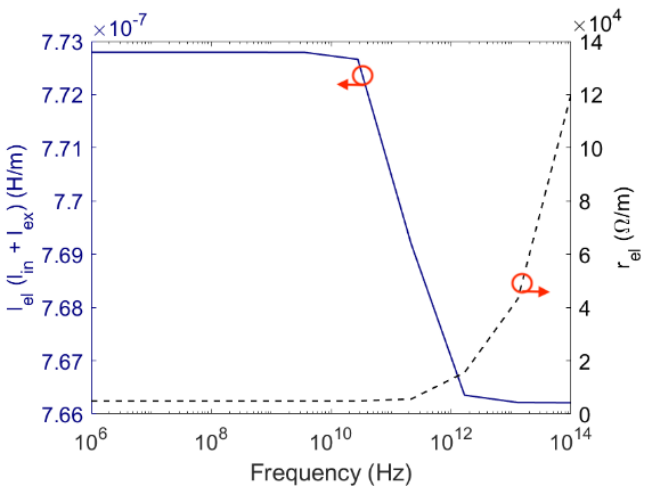

(a)

Figure 8: Simulation results of the impedance (a) and admittance (b) variables of the PZT-IDT finger structure for different frequencies

\section{Simulation of the voltage along the IDT electrodes}

The two coupled differential equations (Eq. 20 and Eq. 21) are evaluated using a solver for ordinary differential equations with known boundary value conditions. If the interval over which the equations are solved is defined as $[0, \mathrm{~L}]$ with $\mathrm{L}$ the length of the electrode finger, the boundary conditions are $V_{1}(x=0)=V_{0}, V_{2}(x=L)=0 V$ and $\frac{d V_{1}}{d y}(x=L)=\frac{d V_{2}}{d y}(x=0)=0$ (the current at end of electrode needs to be zero).

Using the parameters obtained from the in-plane characterization, the differential equations can be solved and the voltage along the electrodes is obtained as shown in Figure 9. As one can see, there is a frequency range in which the voltage at the end of the line is slightly larger than the applied voltage. This occurs for frequencies higher than $22 \mathrm{MHz}$ (up to several $\mathrm{GHz}$ ) and is the result of a resonance along the electrode finger since a similar LCR network has a resonance frequency of about the same value and a damping factor smaller than one. However this voltage increase is very small and in general it can be said that the voltage along the electrode in the $\mathrm{MHz}$ range is constant. For frequencies above $10 \mathrm{GHz}$, a notable voltage drop is observed. 


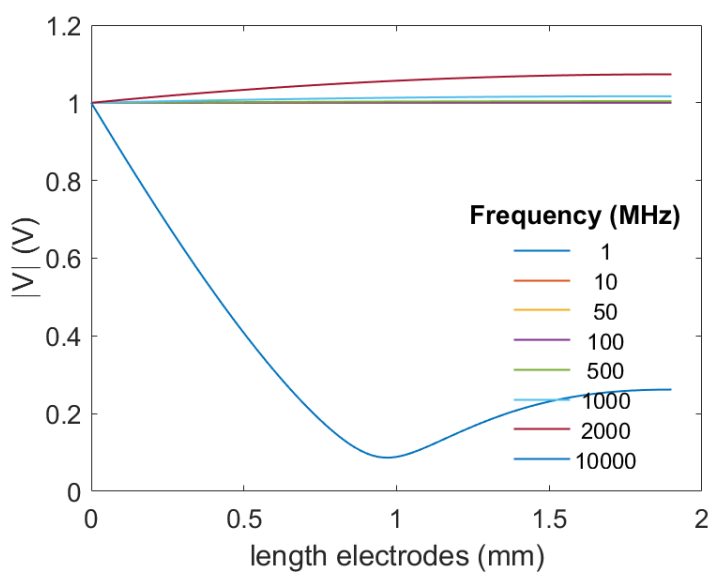

Figure 9: Simulation of the voltage along one IDT electrode finger. For frequencies higher than $22 \mathrm{MHz}, a$ small increase of the voltage is observed. In the $\mathrm{MHz}$ range the voltage along the finger can be assumed to be constant.

Knowing the voltage and impedance values of the electrodes and PZT, the total impedance of the electrode fingers with PZT in between can be calculated. Figure 10 presents the real and imaginary part of the impedance for low frequencies. As expected, the imaginary part of the impedance is negative and in absolute value larger than the real part proving the structure behaves as a capacitor.

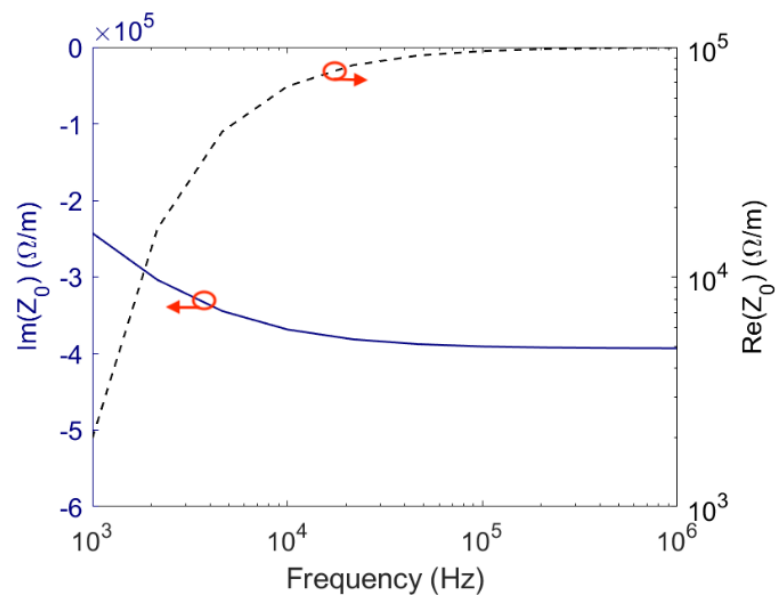

Figure 10: Simulation of the real and imaginary part of the impedance of the electrode fingers with PZT for low frequencies

From the calculation of the voltage along the electrodes at different frequencies, the simulations show that it is possible to determine the impedance of one finger pair, even with the high permittivity of the PZT layer. Knowing this, the model can be extended to calculate the total impedance of the IDT-PZTglass structure.

\section{Total impedance of the IDT-PZT on glass structure}

The total impedance of the IDT-PZT-glass structure can be seen as a ladder network as shown in Figure 7. There are 3 different types of impedances: $Z_{\mathrm{c}}$ for the contact electrodes towards the IDT, $Z_{\mathrm{e}}$ represents the horizontal connecting electrodes between the fingers and $Z_{0}$ the impedance of the electrode fingers with PZT in between. To calculate the last one, half a positive and half a negative electrode are used with the PZT in between, since the capacitance of the IDT structure can be seen as $2 \mathrm{~N}-1$ capacitors in parallel, where $\mathrm{N}$ is the number of finger pairs.

Figure 7 shows the network equivalent of the PZT-IDT finger structure. If $Z_{\mathrm{el}}$ is the impedance of the electrode fingers given by $Z_{e l}=\left(j \omega\left(2 l_{\text {in }}+l_{e x}\right)+r_{e l}\right) L_{f i n g e r}$ and $\mathrm{Y}_{\mathrm{PZT}}$ the admittance equivalent of the effect of the PZT layer in between given by $Y_{P Z T}=(j \omega c+g) L_{\text {aperture }}$, the impedance of this 
section $\mathrm{Z}_{0}=\mathrm{Z}_{\mathrm{el}}+\left(1 / \mathrm{Y}_{\mathrm{PZT}}\right)$. Here the assumption is made that the capacitance between the end of the finger and the bus bar electrodes is much smaller than the one between the two fingers and can be neglected.

Similarly, the inductance of the electrode (bus bar) between 2 fingers $Z_{e}=\left(j \omega l^{\prime}{ }_{i n}+l^{\prime}{ }_{e l}\right) \frac{\lambda_{0}}{2}$ where $l^{\prime}{ }_{\text {in }}$ and $r^{\prime}$ el are p.u.l. values calculated using the thickness and width of the electrodes (here $d_{c}=400 \mathrm{~nm}$, width $=200 \mu \mathrm{m})$. Finally, $Z_{\mathrm{c}}$ represents the long contact electrodes towards the structure and is given by $Z_{c}=\left(j \omega l^{\prime}{ }_{i n}+r_{e l}^{\prime}\right) L_{\text {contacts }}$. For $Z_{\mathrm{c}}$, only the internal inductance is used since the distance between the two contact electrodes is quite large and can be neglected.

Using the ladder network presented in Figure 7, the total impedance of the structure can be calculated by a continued fraction

$$
Z_{\text {tot }}=\left[2 Z_{c} ; \frac{1}{Z_{0}}, 2 Z_{e}, \frac{1}{Z_{0}}, 2 Z_{e}, \ldots, Z_{e}, \frac{1}{Z_{0}}\right]
$$

The total impedance for a $50 \mu \mathrm{m}$ IDT with 20 finger pairs can be calculated using the parameters mentioned earlier. At $100 \mathrm{MHz}$, the total impedance $Z_{\text {tot }}=11.27-72.71 \mathrm{j} \Omega$. Here, the capacitance behaviour of the IDT is the main factor given the negative and large value of the imaginary part.

The calculated impedance for different frequencies, thicknesses of the PZT film and IDT sizes can be compared to impedance measurements which are performed using a LCR tool measuring the capacitance, conductance and admittance (absolute value and phase). Due to the frequency limit of the LCR tool, it is only possible to measure in the kHz-frequency range. Figure 11 shows the measured impedance (absolute real and imaginary part) and the simulated one. As one can see, at these frequencies the measured real part is similar but slightly smaller than the simulated impedance and the measured imaginary value matches the simulation result for frequencies above $100 \mathrm{kHz}$ but overestimates the reactance value for lower frequencies. The close agreement between the simulation and measurements indicates the accuracy of the presented model for frequencies above $100 \mathrm{kHz}$.

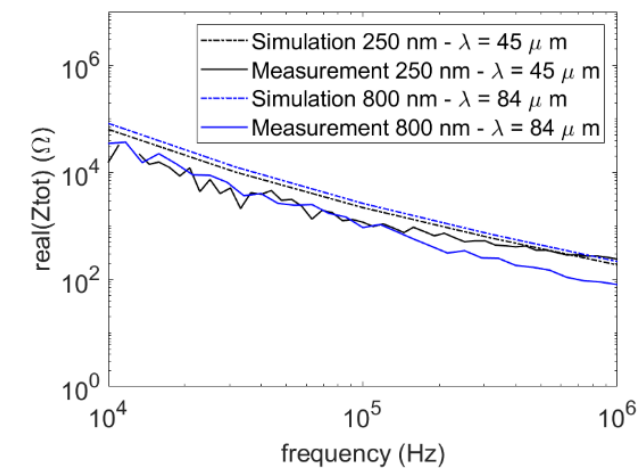

(a)

Figure 11: Comparison simulated and measured impedance (a) real part and (b) imaginary part of $Z_{\text {tot }}$

\section{Conclusion}

We have shown that in-plane dielectric characterization is possible using IDT electrodes. The dielectric constant obtained from FEM simulations and low frequency capacitance measurements is in agreement with the value obtained from hysteresis measurements and is about 1000 for $440 \mathrm{~nm}$ thick PZT films but increases for thinner films. Hysteresis measurements indicate the switching of the polarization of the ferroelectric domains, showing the possibility to pole the ferroelectric PZT film in-plane. Furthermore, the low leakage current measured during steady state IV measurements indicates the high 
quality of the film. Using the measured characteristics, we were able to develop a general impedance model of the IDT-PZT structure. Comparing the obtained admittance of this model to low frequency measurements gives an excellent fit to the measurement data.

\section{Acknowledgments}

Tessa Van de Veire acknowledges support and funding as $\mathrm{PhD}$ grant strategic basic research of the Research Foundation - Flanders (FWO) under grant number 1SC3919N. Hannes Rijckaert acknowledges support and funding as postdoctoral fellow fundamental research of the Research Foundation - Flanders (FWO) under grant number 1273621N.

\section{Data availability}

The data that support the findings of this study are available from the corresponding author upon reasonable request.

\section{References}

[1] K. Alexander et al., "Nanophotonic Pockels modulators on a silicon nitride platform," pp. 1$11,2018$.

[2] P. Castera, D. Tulli, A. M. Gutierrez, and P. Sanchis, "Influence of BaTiO_ 3 ferroelectric orientation for electro-optic modulation on silicon," Opt. Express, vol. 23, no. 12, p. 15332, 2015.

[3] K. Amanuma et al., "Characteristics of $0.25 \mu \mathrm{m}$ ferroelectric nonvolatile memory with a $\mathrm{Pb}(\mathrm{Zr}$, Ti)O3 capacitor on a metal/via-stacked plug," Japanese J. Appl. Physics, Part 1 Regul. Pap. Short Notes Rev. Pap., vol. 39, no. 4 B, pp. 2098-2101, 2000.

[4] Y. Wang, W. Chen, B. Wang, and Y. Zheng, "Ultrathin ferroelectric films: Growth, characterization, physics and applications," Materials (Basel)., vol. 6, no. 9, pp. 6377-6485, 2014.

[5] N. R. Harris et al., "A multilayer thick-film PZT actuator for MEMs applications," Sensors Actuators, A Phys., vol. 132, no. 1 SPEC. ISS., pp. 311-316, 2006.

[6] S. Trolier-McKinstry and P. Muralt, "Thin Film Piezoelectrics for MEMS," J. Electrceramics, vol. 12, pp. 7-17, 2004.

[7] K. Länge, B. E. Rapp, and M. Rapp, "Surface acoustic wave biosensors: A review," Anal. Bioanal. Chem., vol. 391, no. 5, pp. 1509-1519, 2008.

[8] N. Izyumskaya et al., "Processing, Structure, Properties, and Applications of PZT Thin Films," Crit. Rev. Solid State Mater. Sci., vol. 32, no. September, pp. 111-202, 2007.

[9] R. Gupta, V. Gupta, and M. Tomar, "Ferroelectric PZT thin films for photovoltaic application," Mater. Sci. Semicond. Process., vol. 105, no. September 2019, p. 104723, 2020.

[10] R. Weigel et al., "Microwave Acoustic Materials, Devices , and Applications," IEEE Trans. Microw. Theory Tech., vol. 50, no. 3, pp. 738-749, 2002.

[11] J. Friend and L. Yeo, "Piezoelectric Materials for Microfluidics," in Encyclopedia of Microfluidics and Nanofluidics, D. Li, Ed. Boston, MA: Springer US, 2008, pp. 1654-1662.

[12] C. B. Eom and S. Trolier-McKinstry, "Thin-film piezoelectric MEMS," MRS Bull., vol. 37, no. 11, pp. 1007-1017, 2012.

[13] M. M. Zhu, Z. H. Du, and J. Ma, "Influence of crystal phase and transparent substrates on electro-optic properties of lead zirconate titanate films," J. Appl. Phys., vol. 108, no. 11, p. 113119, Dec. 2010. 
[14] Y. Q. Fu et al., "Advances in piezoelectric thin films for acoustic biosensors, acoustofluidics and lab-on-chip applications," Prog. Mater. Sci., vol. 89, pp. 31-91, 2017.

[15] E. Sun and W. Cao, "Relaxor-based ferroelectric single crystals: Growth, domain engineering, characterization and applications," Prog. Mater. Sci., vol. 65, pp. 124-210, 2014.

[16] J. P. George et al., "Lanthanide-Assisted Deposition of Strongly Electro-optic PZT Thin Films on Silicon: Toward Integrated Active Nanophotonic Devices," ACS Appl. Mater. Interfaces, vol. 7, no. 24, pp. 13350-13359, 2015.

[17] R. R. Khan and S. Kang, "Highly Sensitive Multi-Channel IDC Sensor Array for Low Concentration Taste Detection," Sensors, vol. 15, pp. 13201-13221, 2015.

[18] V. H. Nguyen, O. Peters, S. Serve, and U. Schnakenberg, "Portable SAW Impedance Sensor Using a 1-Port Resonator Approach," Proceedings, vol. 1, no. 4, pp. 1-4, 2017.

[19] W. C. Wilson and G. M. Atkinson, "Rapid SAW Sensor Development Tools," 2019.

[20] R. Nigon, T. M. Raeder, and P. Muralt, "Characterization methodology for lead zirconate titanate thin films with interdigitated electrode structures," J. Appl. Phys., vol. 204101, pp. 012, 2017.

[21] C. H. Nguyen, R. Nigon, T. M. Raeder, and U. Hanke, "Probing-models for interdigitated electrode systems with ferroelectric thin films," J. Phys. D. Appl. Phys., vol. 51, 2018.

[22] N. Chidambaram, A. Mazzalai, D. Balma, and P. Muralt, "Comparison of Lead Zirconate Titanate Thin Films for Microelectromechanical Energy Harvester With Interdigitated and Parallel Plate Electrodes," IEEE Trans. Ultrason. Ferroelectr. Freq. Control, vol. 60, no. 8, pp. 1564-1571, 2013.

[23] R. Bouregba and G. Poullain, "Numerical Extraction of the True Ferroelectric Polarization Due to Switching Domains from Hysteresis Loops Measured Using A Sawyer-Tower Circuit," Ferroelectrics, vol. 274, no. January 2002, pp. 165-181, 2015.

[24] L. Pintilie, I. Vrejoiu, D. Hesse, and M. Alexe, "The influence of the top-contact metal on the ferroelectric properties of epitaxial ferroelectric $\mathrm{Pb}(\mathrm{Zr} 0.2 \mathrm{Ti0} .8) \mathrm{O} 3$ thin films," J. Appl. Phys., vol. 104, no. 11, pp. 0-6, 2008.

[25] J. Cheng et al., "Dielectric properties of (100) textured thick $\mathrm{Pb}(\mathrm{Zr} x \mathrm{Ti} 1-\mathrm{x}) \mathrm{O} 3$ films with different Zr/Ti atom ratios," J. Appl. Phys., vol. 5997, no. 100, 2002.

[26] H. Kozuka, "Stress evolution and cracking in sol-gel-derived thin films," in Handbook of SolGel Science and Technology: Processing, Characterization and Applications, 2018, pp. 275311.

[27] R. Bouregba, B. Vilquin, G. Le Rhun, and G. Poullain, "Sawyer - Tower hysteresis measurements on micron sized $\mathrm{Pb}$ ( Zr , Ti ) O3 capacitors," Rev. Sci. Instrum., vol. 74, no. October, pp. 4429-4435, 2003.

[28] N. Chidambaram et al., "Converse mode piezoelectric coefficient for lead zirconate titanate thin film with interdigitated electrode," J. Micromechanics Microengineering, vol. 25, 2015.

[29] Y. Zhou, H. K. Chan, C. H. Lam, and F. G. Shin, "Mechanisms of imprint effect on ferroelectric thin films," J. Appl. Phys., vol. 98, no. 2, p. 024111, 2005.

[30] L. Pintilie and M. Alexe, "Metal-ferroelectric-metal heterostructures with Schottky contacts. I. Influence of the ferroelectric properties," J. Appl. Phys., vol. 98, no. 12, 2005.

[31] F. Chen et al., "Barrier heights, polarization switching, and electrical fatigue in $\mathrm{Pb}(\mathrm{Zr}, \mathrm{Ti}) \mathrm{O} 3$ ceramics with different electrodes," J. Appl. Phys., vol. 108, no. 10, p. 104106, Nov. 2010. 
[32] D. G. Popescu, M. A. Husanu, C. Chirila, L. Pintilie, and C. M. Teodorescu, "Impact on Ferroelectricity and Band Alignment of Gradually Grown Au on $\mathrm{BaTiO}_{3}$," Phys. status solidiRapid Res. Lett., vol. 13, no. 7, p. 1900077, Jul. 2019.

[33] N. G. Apostol et al., "Band bending at copper and gold interfaces with ferroelectric $\mathrm{Pb}(\mathrm{Zr}, \mathrm{Ti}) \mathrm{O}$ 3 investigated by photoelectron spectroscopy," Appl. Surf. Sci., vol. 354, pp. 459-468, Nov. 2015.

[34] J. K. Sinha, "Modified Sawyer and Tower circuit for the investigation of ferroelectric samples," J. Sci. Instrum, vol. 42, p. 696, 1965.

[35] C. L. Holloway, S. Member, and E. F. Kuester, "DC Internal Inductance for a Conductor of Rectangular Cross Section,” pp. 1-7, 2009.

[36] C. R. Paul, Analysis of multiconductor transmission lines, Second edi. John Wiley \& Sons, 2008.

[37] C. L. Holloway, E. F. Kuester, A. E. Ruehli, and L. Fellow, "Partial and Internal Inductance : Two of Clayton R. Paul' s Many Passions," IEEE Trans. Electromagn. Compat., vol. 55, no. 4, pp. 600-613, 2013.

[38] M. Matsuki and A. Matsushima, "Efficient Impedance Coputation For Multi-conductor Transmission Lines of Rectangular Cross Section," Prog. Electromagn. Res. B, vol. 43, no. July, pp. 373-391, 2012.

[39] G. Antonini, A. Orlandi, S. Member, and C. R. Paul, "Internal Impedance of Conductors of Rectangular Cross Section," IEEE Trans. Microw. Theory Tech., vol. 47, no. 1, pp. 979-985, 1999. 


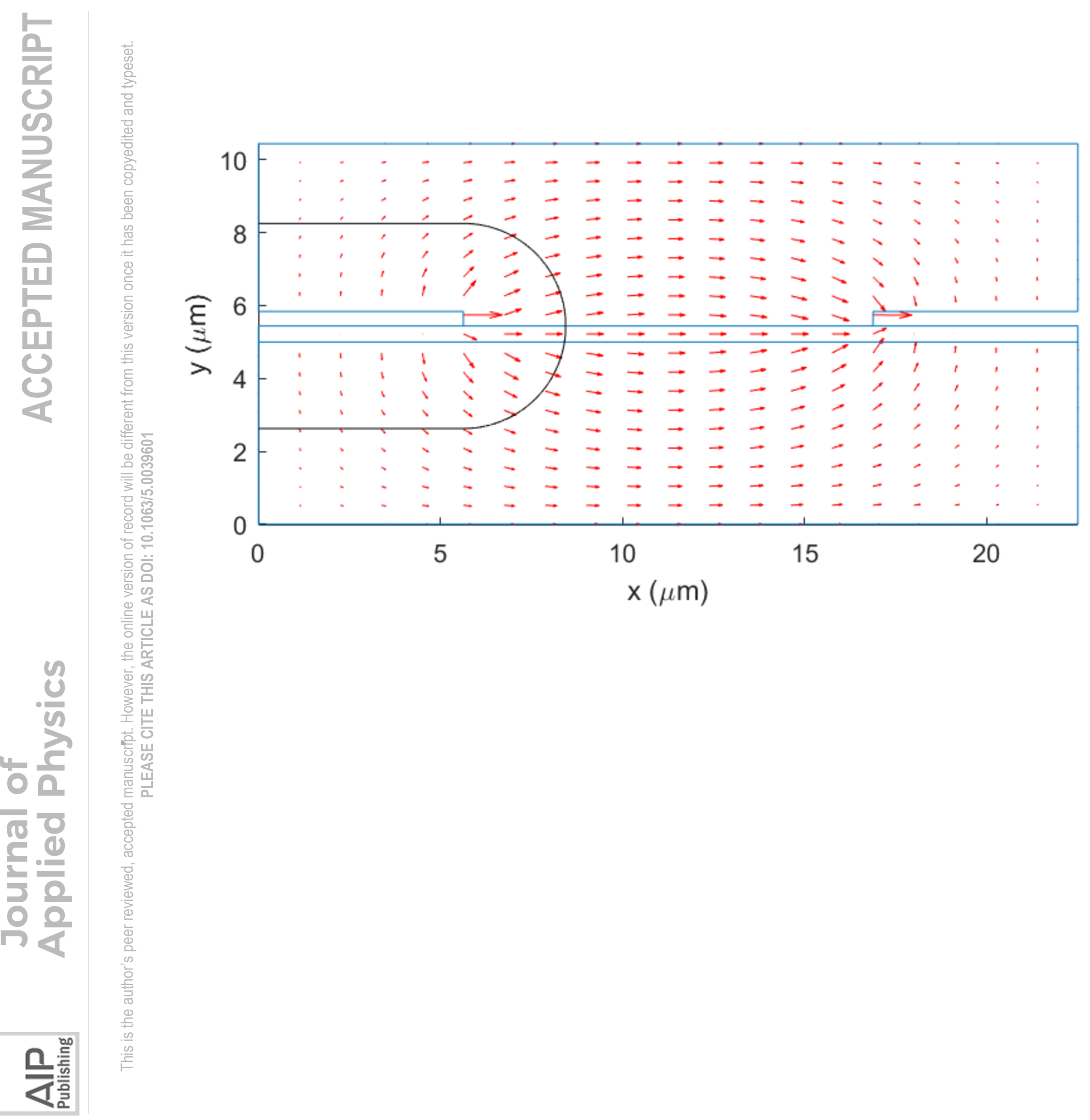



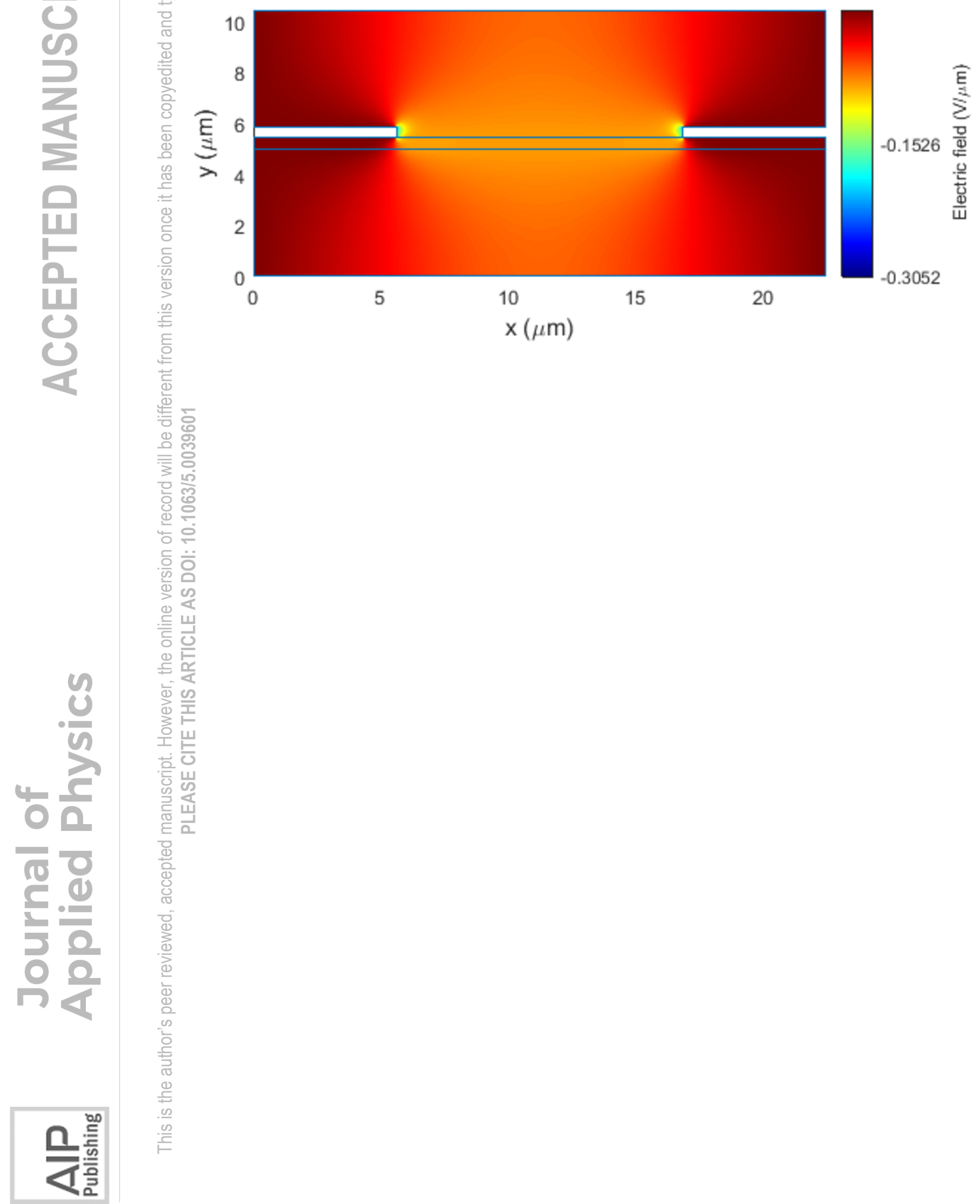

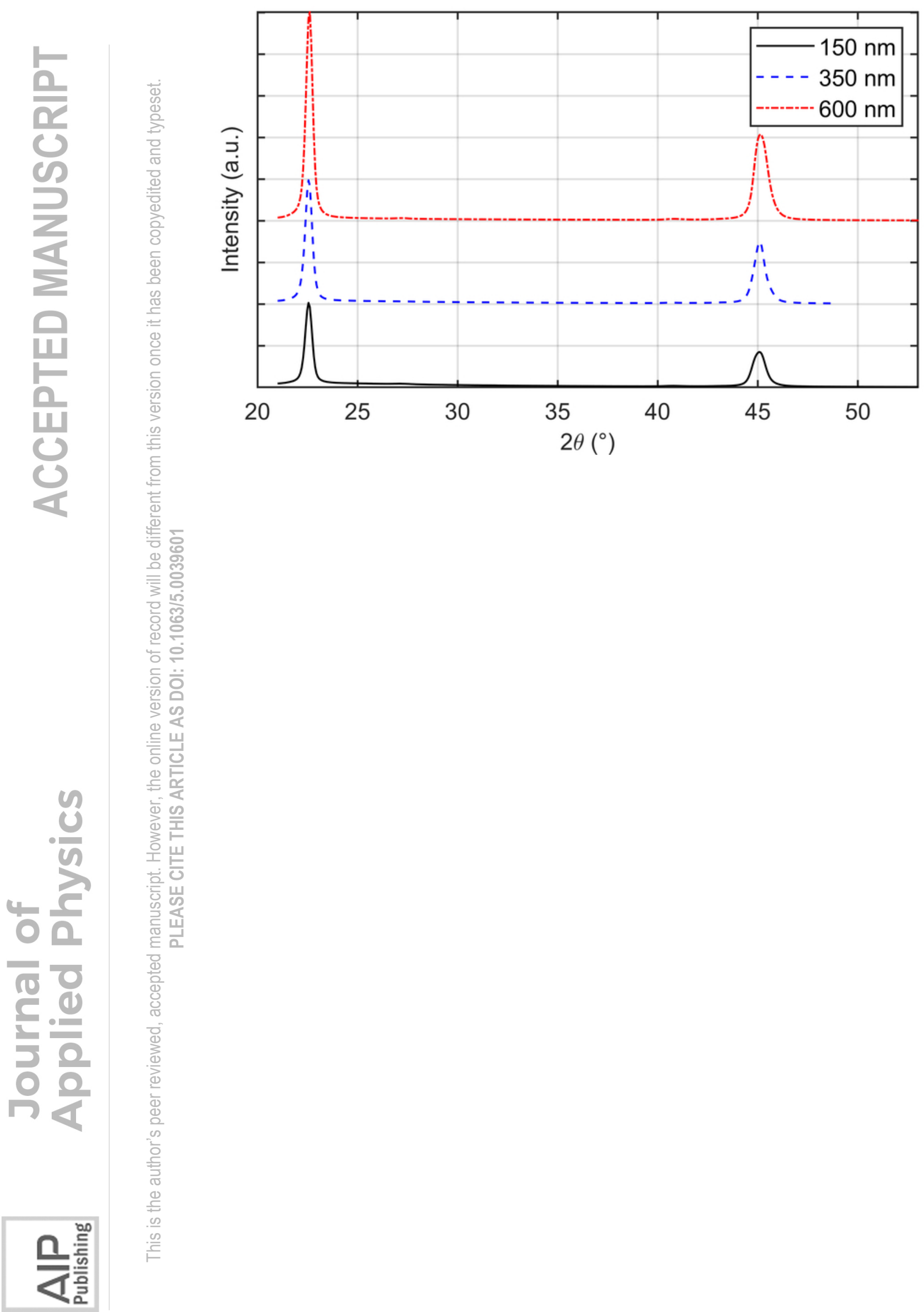

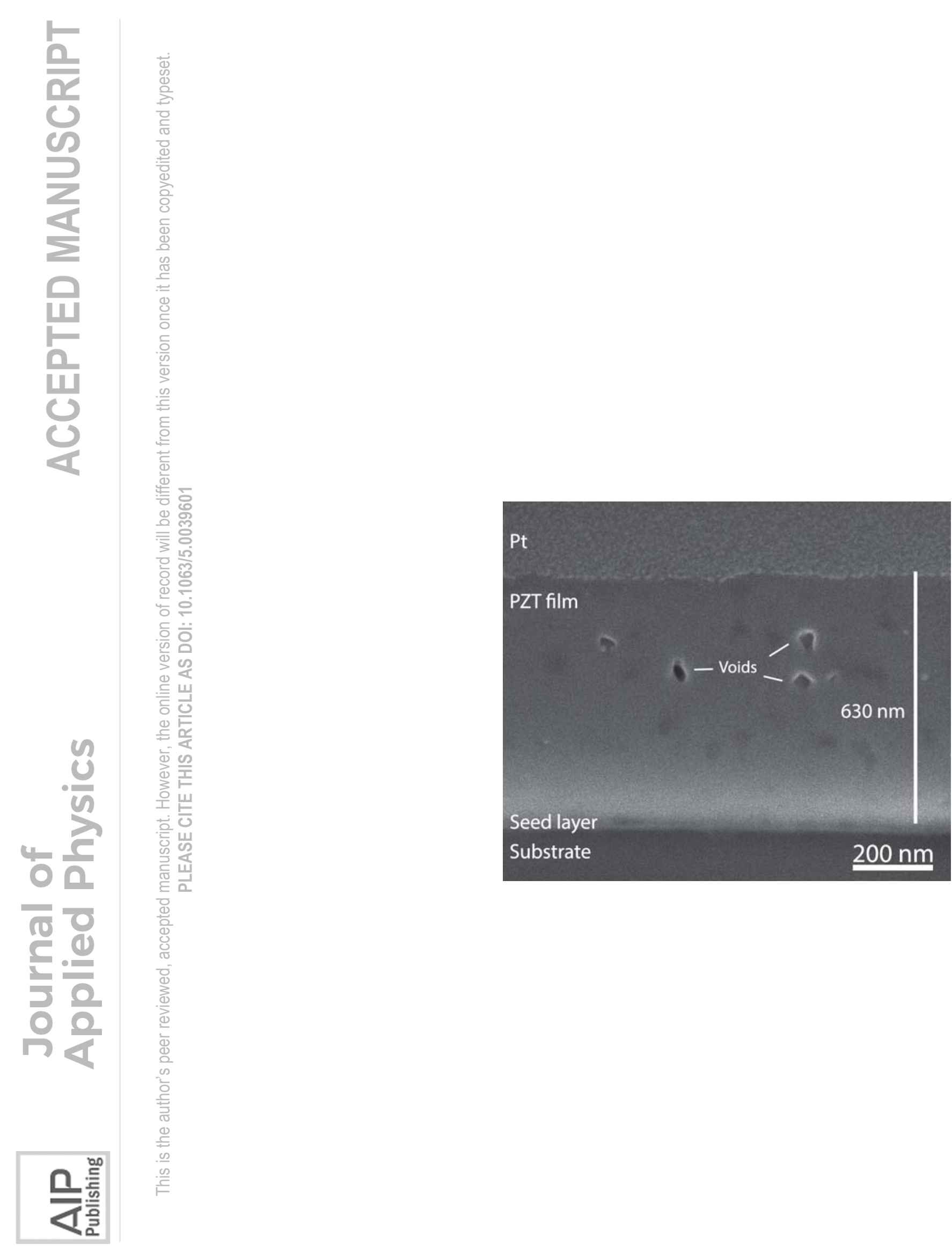


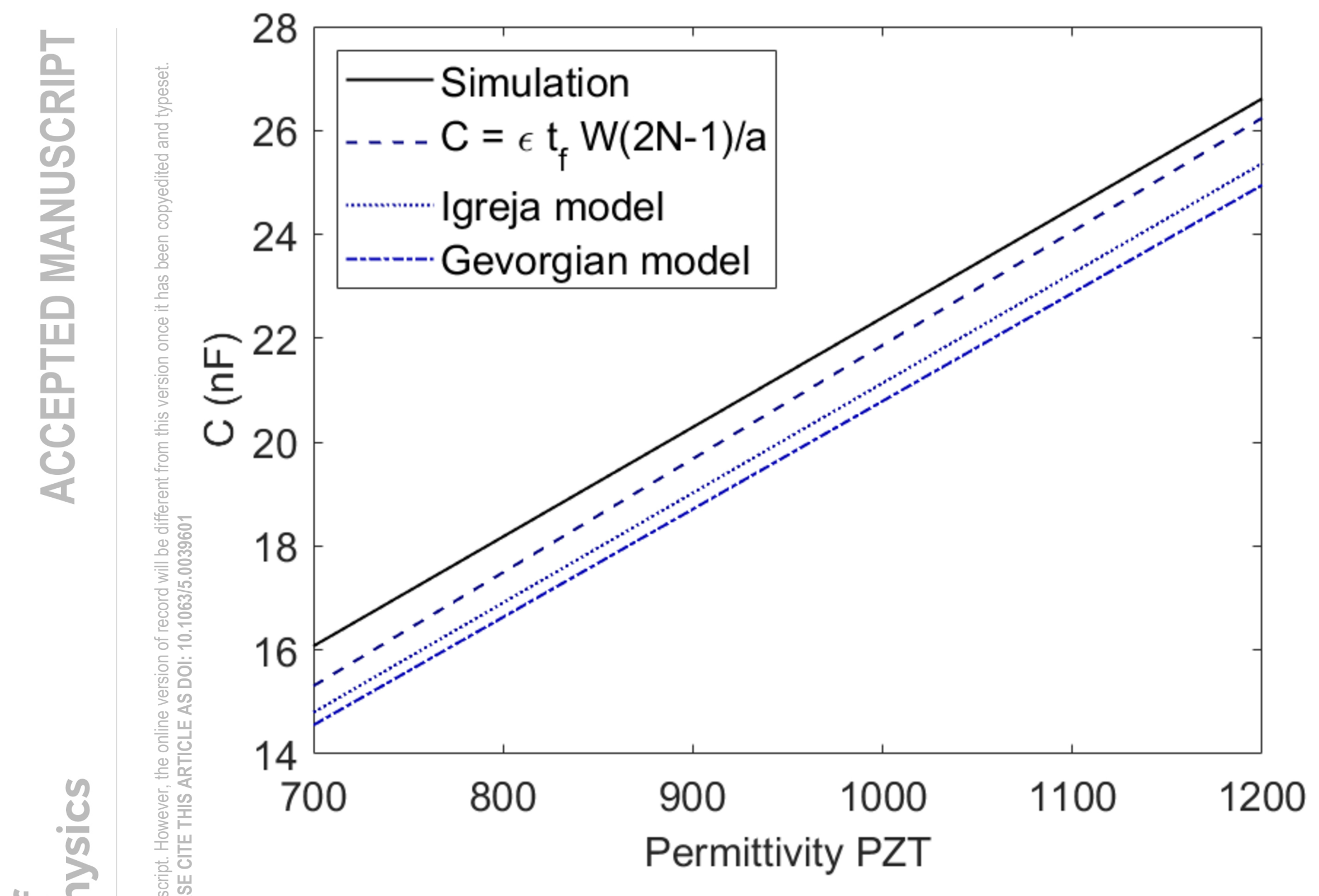

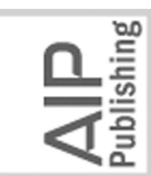




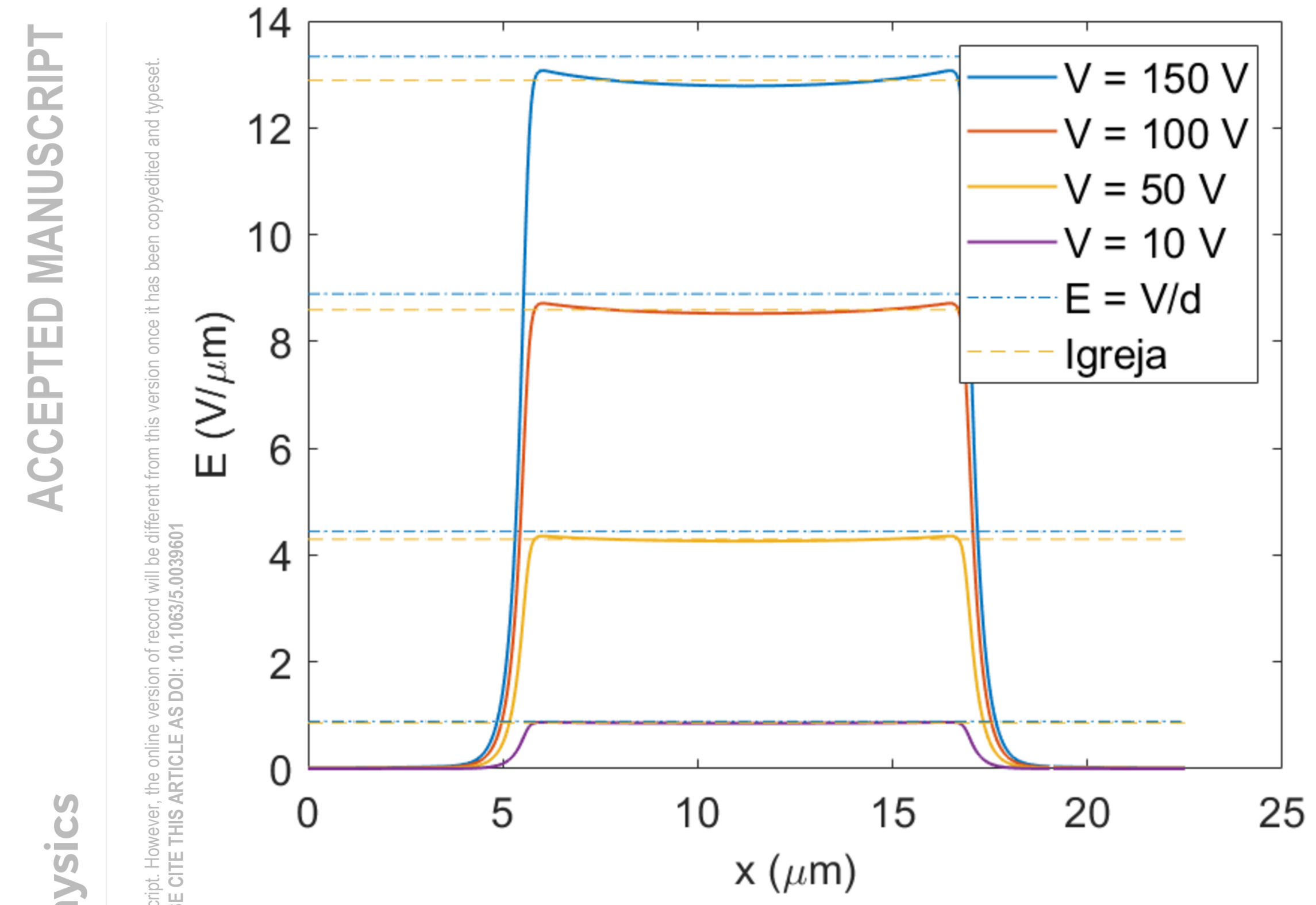




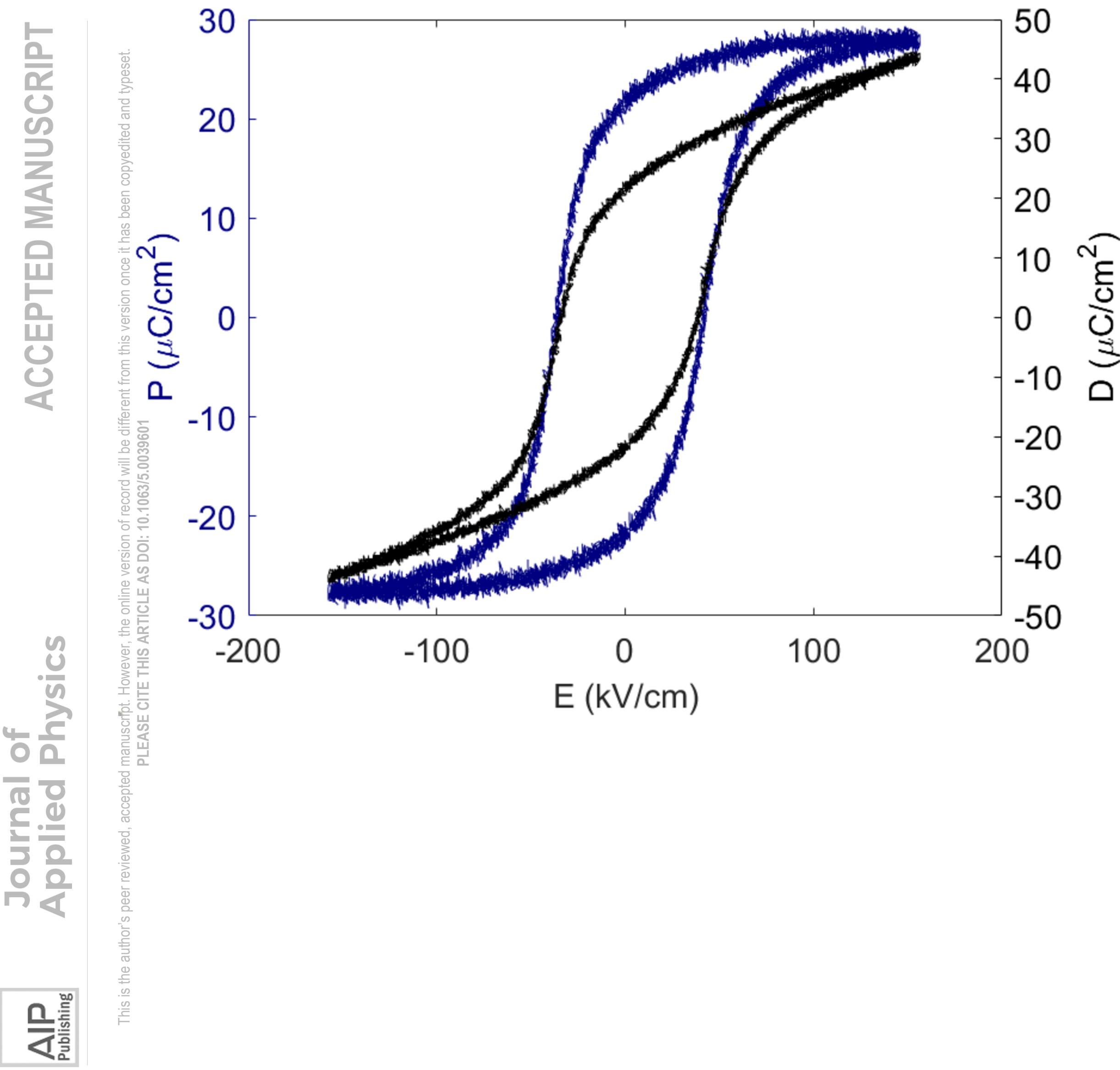




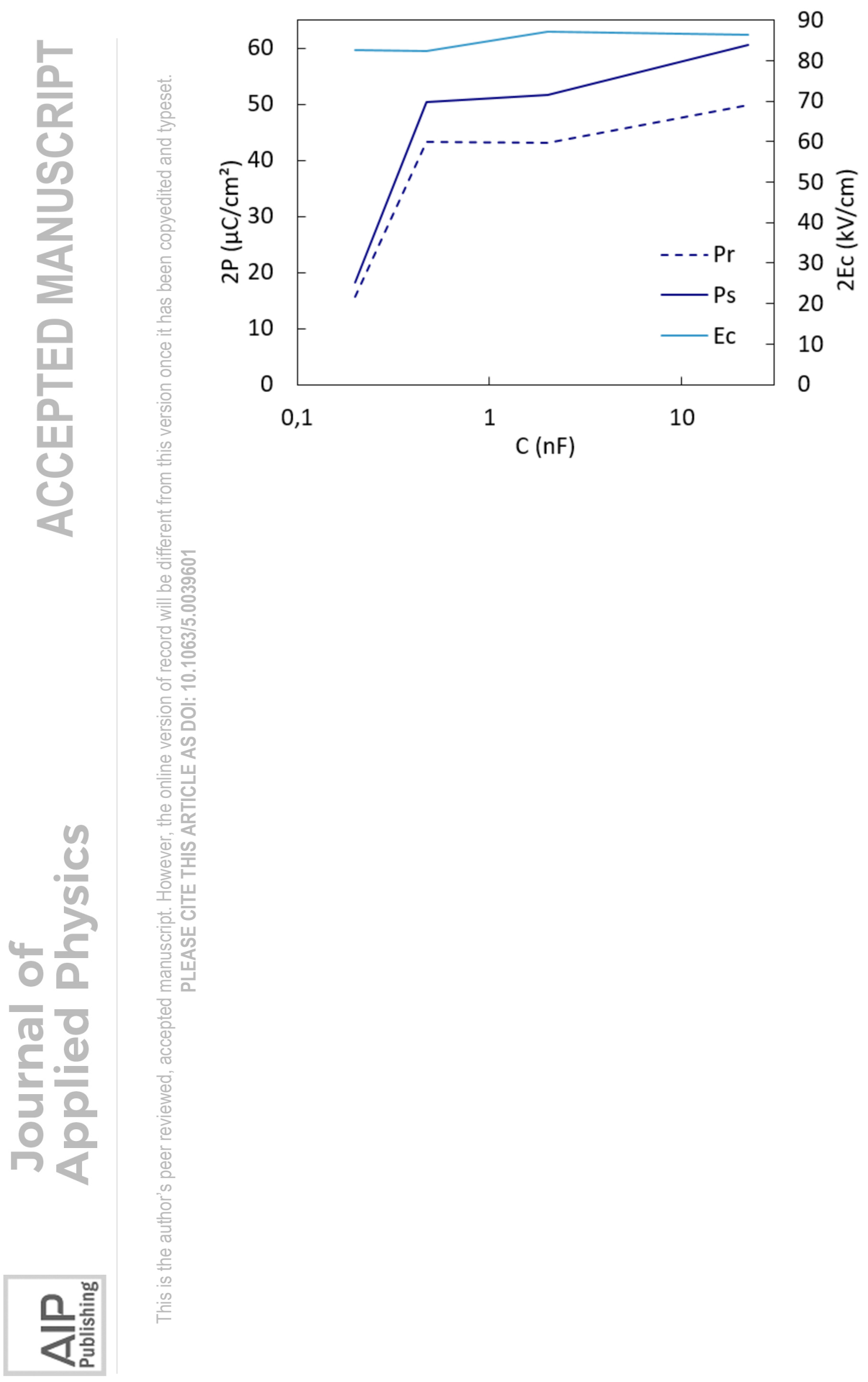




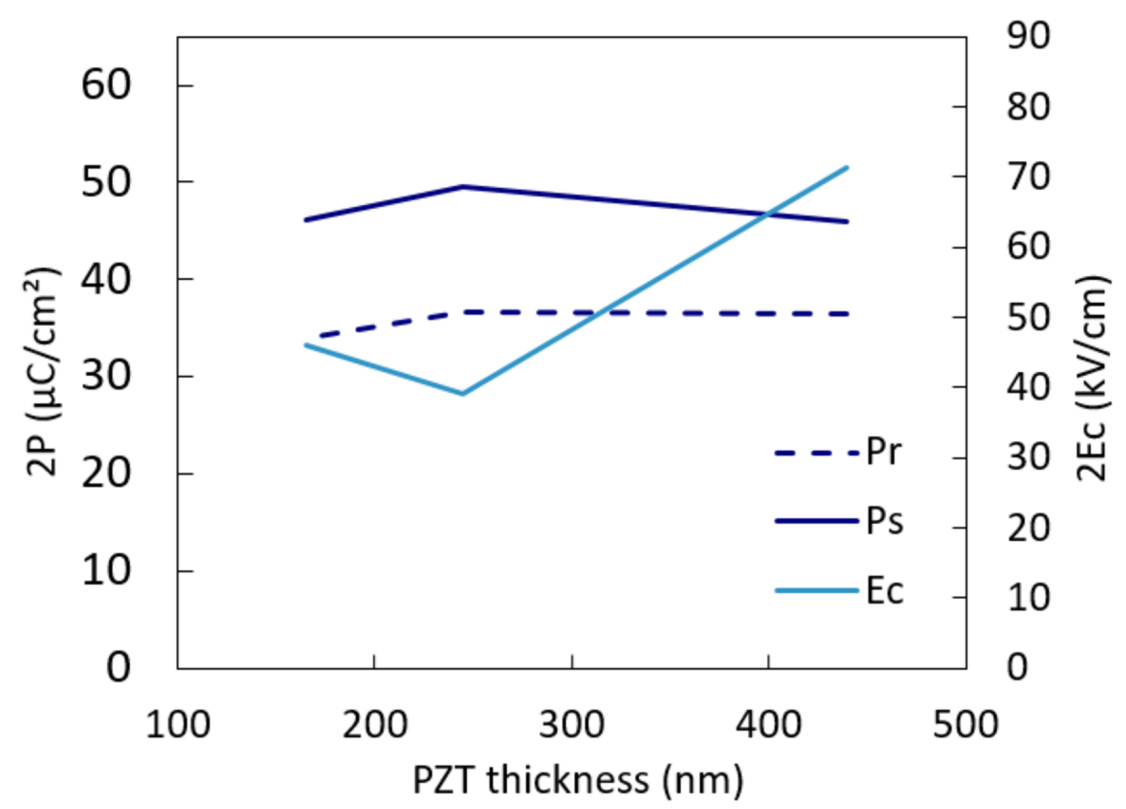




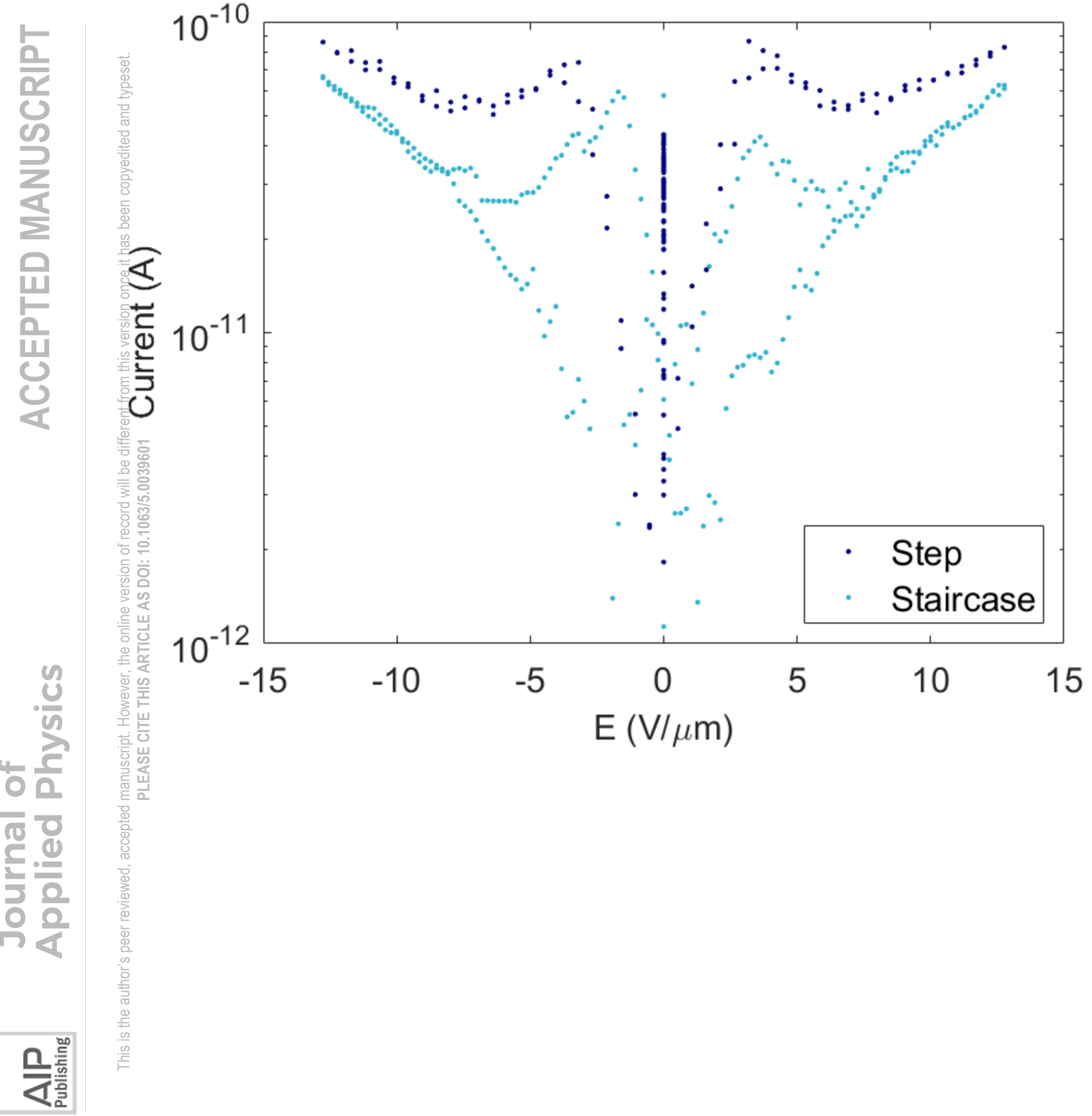




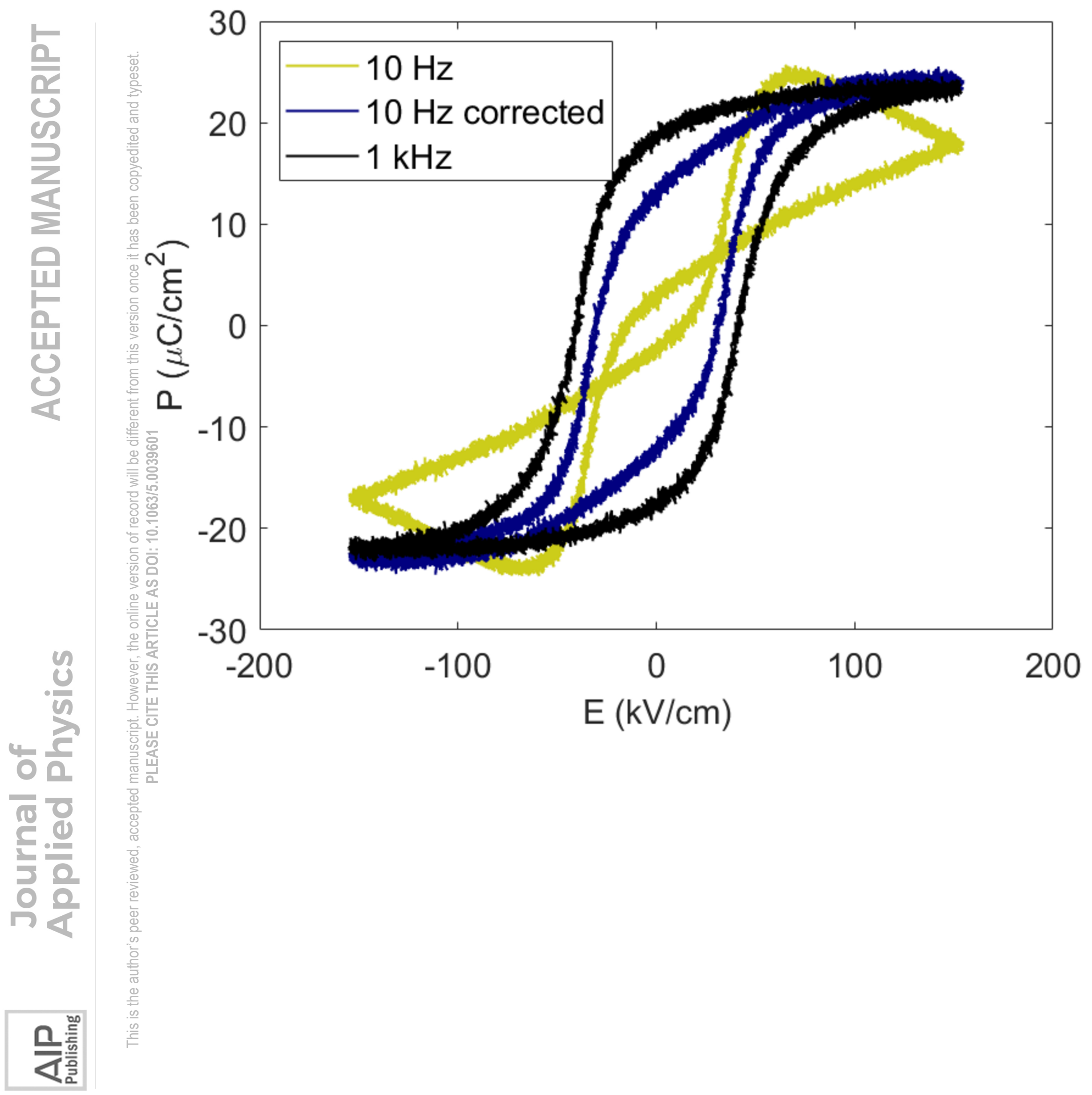




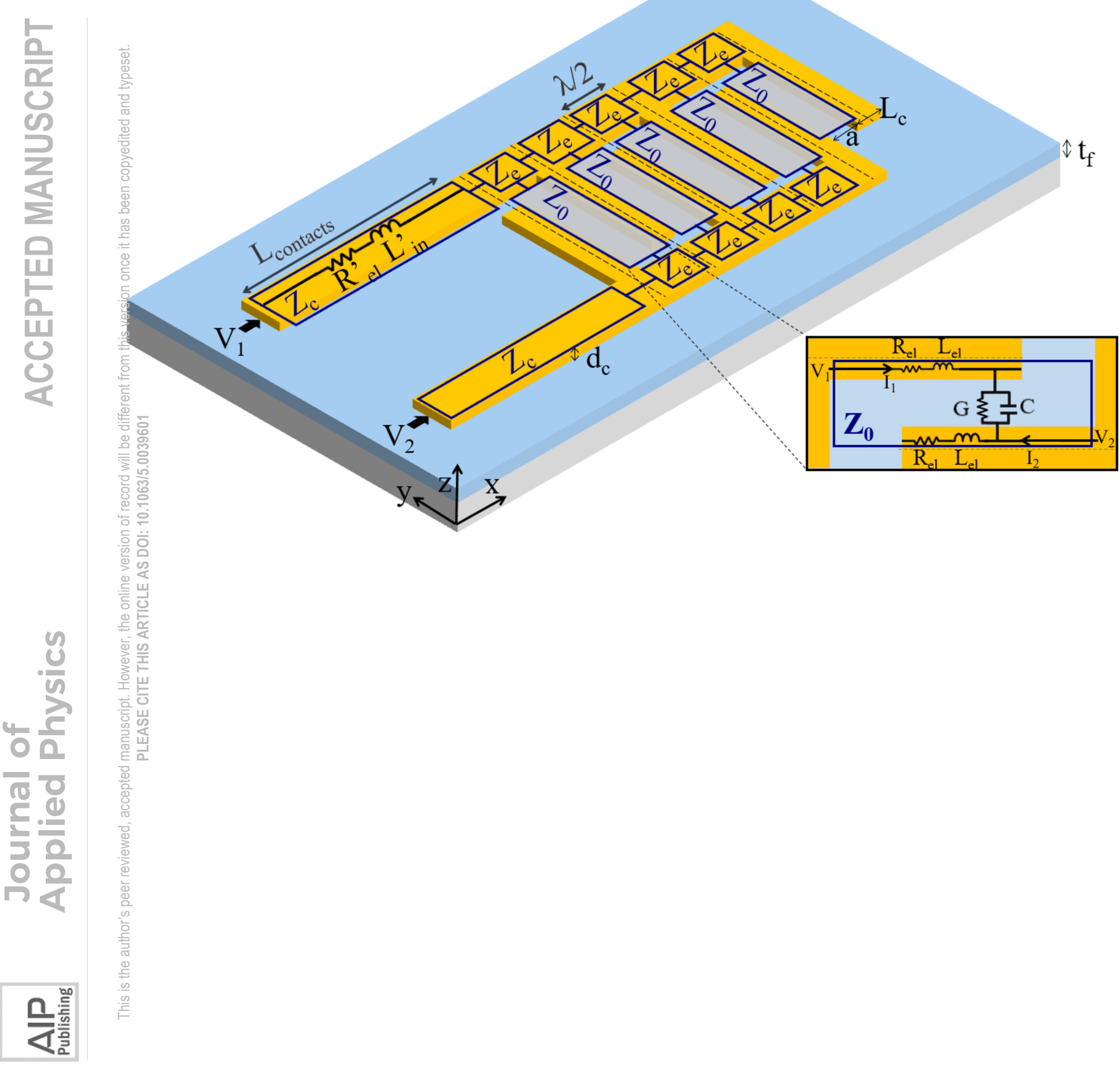




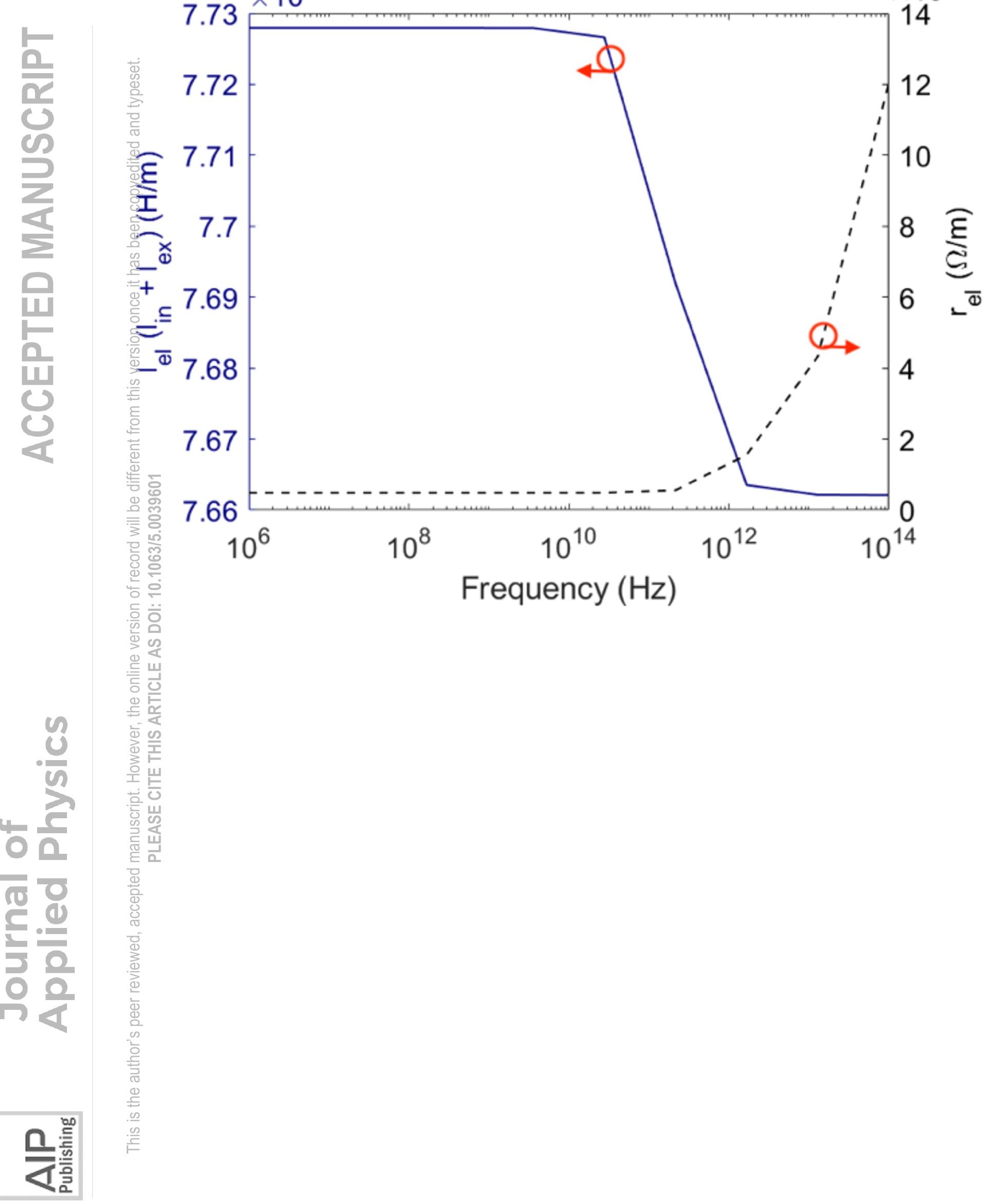




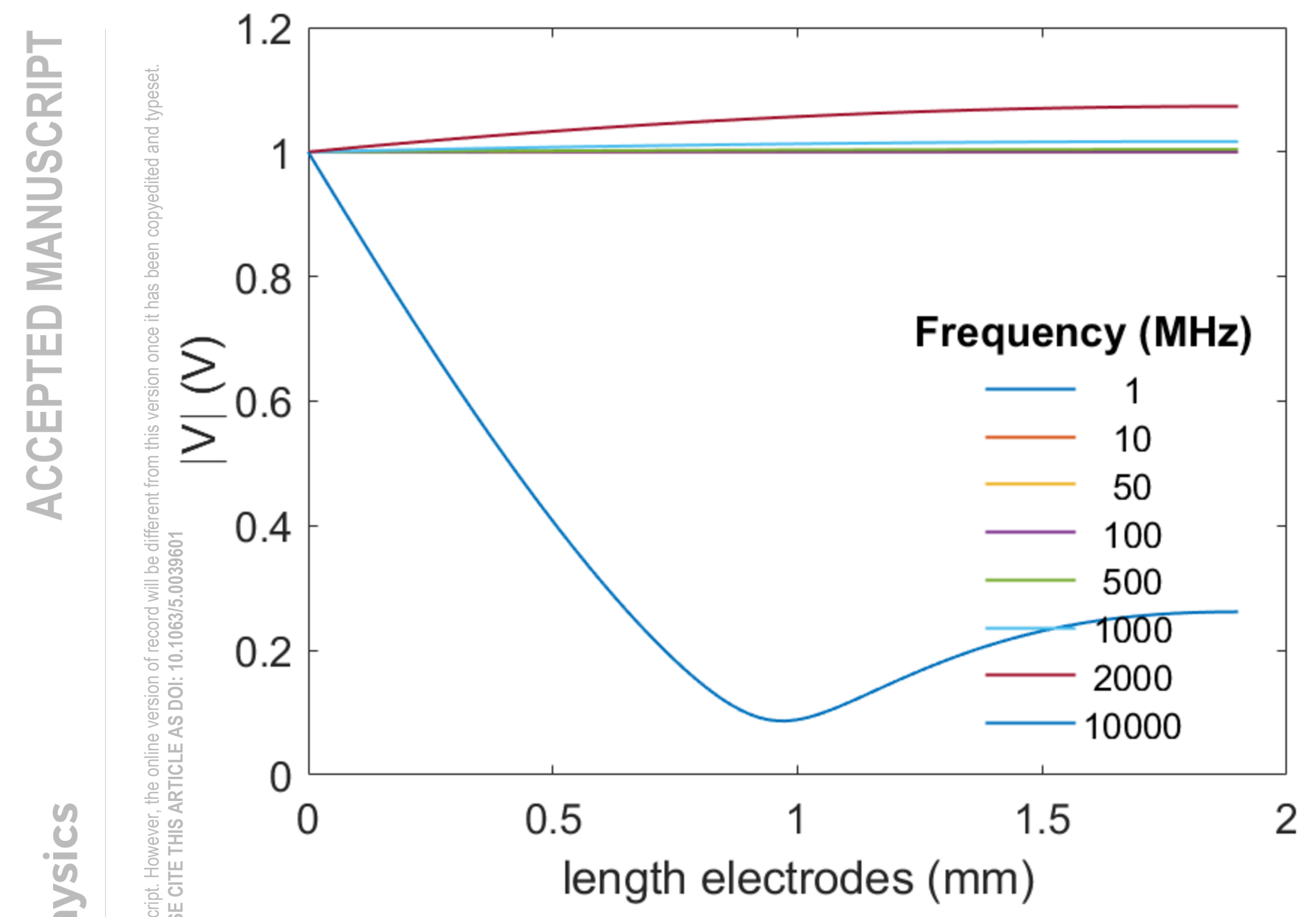




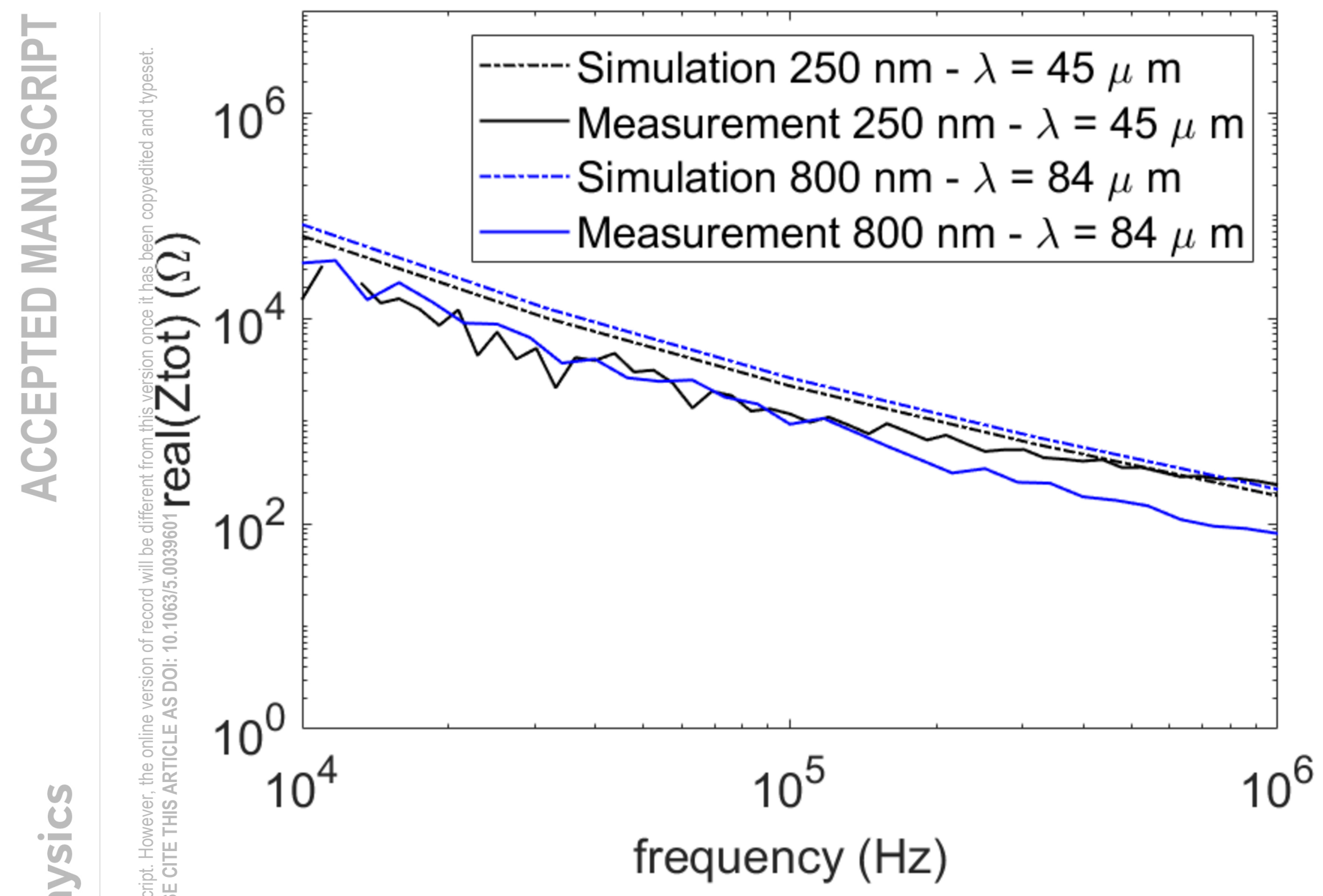


